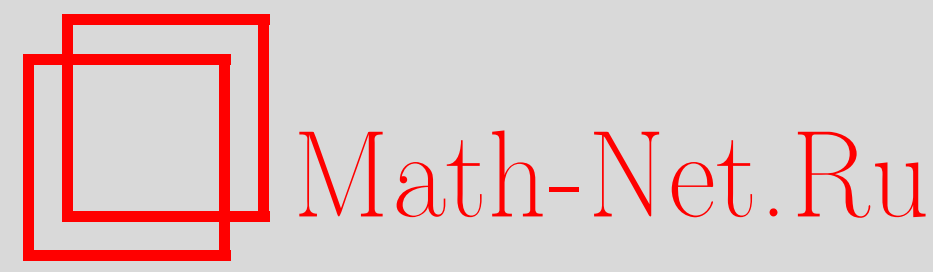

В. П. Михайлов, О существовании граничных значений у метагармонических функций, Матем. сб., 1999, том 190, номер 10, 17-48

DOI: https://doi.org/10.4213/sm431

Использование Общероссийского математического портала Math-Net.Ru подразумевает, что вы прочитали и согласны с пользовательским соглашением

http://www . mathnet.ru/rus/agreement

Параметры загрузки:

IP: 34.227 .88 .159

26 апреля 2023 г., $14: 38: 36$ 
УДК 517.9

\author{
В.П. Михайлов
}

\title{
О существовании граничных значений у метагармонических функций
}

\begin{abstract}
Установлены необходимые и достаточные условия существования $L_{2}$-пределов на границе у решений метагармонического уравнения в полупространстве. Для уравнения Лапласа эти критерии совпадают с теоремой Рисса: гармоническая функция имеет $L_{2}$-предел на границе тогда и только тогда, когда она $L_{2}$-ограничена.
\end{abstract}

Библиографияя: 4 названия.

В работе рассматривается вопрос о существовании предельных значений на граничных поверхностях коразмерности единица у решений эллиптических уравнений. Двумерный случай полигармонических функций в круге с этой точки зрения был рассмотрен в [1]; в этой работе были получены необходимые и достаточные условия для того, чтобы полигармоническая функция имела предел в среднем на граничной окружности. Цель настоящей работы - перенести полученные в [1] критерии на более общий случай метагармонических функций, причем рассматриваться эти функции будут в полупространстве произвольной размерности. План изложения в предлагаемой работе в основном такой же, как и в работе [1], однако реализация этого плана здесь, естественно, встречается с бо́льшими трудностями.

Предельные значения на границе, как и в [1], будут пониматься в смысле $L_{2}$-пределов. При этом решения естественно считать заданньми не во всей области, а лишь в некоторой соответствующей приграничной полосе.

В полосе $\Pi=\left\{x=\left(x_{1}, \ldots, x_{n}\right) \in \mathbb{R}_{n}, 0<y<1\right\}(n+1)$-мерного пространства $\mathbb{R}_{n+1}=\left\{x \in \mathbb{R}_{n}, y \in \mathbb{R}_{1}\right\}$ рассмотрим дифференциальное уравнение

$$
\mathscr{P}(\Delta) u(x, y)=0, \quad(x, y) \in \Pi,
$$

где $\Delta=\frac{\partial^{2}}{\partial x_{1}^{2}}+\cdots+\frac{\partial^{2}}{\partial x_{n}^{2}}+\frac{\partial^{2}}{\partial y^{2}}-$ оператор Лапласа, а

$$
\mathscr{P}(z)=z^{m}+a_{1} z^{m-1}+\cdots+a_{m}
$$

- многочлен (с комплексными коэффициентами) степени $m \geqslant 1$. Уравнение (1), очевидно, является эллиптическим уравнением $2 m$-го порядка, решения его называются метагармоническими функциями [2]. Для простоты мы ограничимся рассмотрением лишь тех решений уравнения (1), которые при любом $a \in(0,1)$ принадлежат пространству $W_{2}^{2 m}\left(\Pi_{a}\right)$, где $\Pi_{a}=\left\{x \in \mathbb{R}_{n}, a<y<1\right\}$, и тем самьм являются непрерывными по $y \in(0,1]$ функциями со значениями в $L_{2}\left(\mathbb{R}_{n}\right)$ : $u(x, y) \in C\left((0,1] ; L_{2}\left(\mathbb{R}_{n}\right)\right)$.

Работа вьполнена при поддержке Российского фонда фундаментальных исследований (грант № 98-01-00248). 
Как обычно, функцию $u(x, y)$ назовем $L_{2}$-ограниченной, если

$$
\sup _{0<y<1}\|u(\cdot, y)\|_{L_{2}\left(\mathbb{R}_{n}\right)}<\infty ;
$$

аналогично, будем говорить, что функция $u(x, y)$ имеет $L_{2}$-предел при $y \rightarrow+0$, если существует функция $u_{0}(x) \in L_{2}\left(\mathbb{R}_{n}\right)$ такая, что

$$
\left\|u(\cdot, y)-u_{0}(\cdot)\right\|_{L_{2}\left(\mathbb{R}_{n}\right)} \rightarrow 0 \text { при } y \rightarrow+0 .
$$

Нас интересует вопрос: какие условия следует наложить на решение $u(x, y)$, чтобы у него существовал $L_{2}$-предел при $y \rightarrow+0$ или иначе, когда $u(x, y) \in$ $C\left([0,1] ; L_{2}\left(\mathbb{R}_{n}\right)\right)$ ?

Для решений уравнения второго порядка известны следующие необходимые и достаточные условия существования $L_{2}$-пределов на границе. Прежде всего это критерий типа теоремы $\Phi$. Рисса: для того чтобы решение $u(x, y)$ уравнения $(1)$ при $m=1$ имело $L_{2}$-предел при $y \rightarrow+0$, необходимо и достаточно, чтобы это решение было $L_{2}$-ограниченным.

Другим критерием является теорема типа теоремы Литлвуда-Пэли: для того чтобы решение $u(x, y)$ уравнения (1) при $m=1$ имело $L_{2}$-предел при $y \rightarrow+0$, необходимо и достаточно, чтобы выполнялось условие

$$
\int_{\Pi}|\nabla u(x, y)|^{2} y d x d y<\infty .
$$

Однако ни условие (2), ни условие (3) не являются критериями в случае, когда уравнение (1) более высокого порядка, $m>1$. Условие $L_{2}$-ограниченности $(2)$, конечно, является необходимым условием сушествования $L_{2}$-предела и при $m>1$, но достаточным условием оно не является. Приведем соответствуюший пример для случая полигармонического уравнения

$$
\Delta^{m} u(x, y)=0, \quad(x, y) \in \Pi, \quad m>1
$$

(в (1) $\left.\mathscr{P}(z)=z^{m}\right)$.

Функция

$$
u(x, y)=\frac{y}{(2 \pi)^{n / 2}} \int_{\mathbb{R}_{n}} e^{i(x, \xi)} \frac{e^{-y|\xi|}}{\left(1+|\xi|^{2}\right)^{(n-2) / 4}} d \xi, \quad(x, y) \in \Pi,
$$

как нетрудно проверить, является бигармонической функцией, т.е. решением уравнения (4) при $m=2$, а значит, и при любом $m \geqslant 2$. Эта функция $L_{2}$-ограничена. Действительно, поскольку

$$
\begin{aligned}
\|u(\cdot, y)\|_{L_{2}\left(\mathbb{R}_{n}\right)}^{2} & =y^{2} \int_{\mathbb{R}_{n}} \frac{e^{-2 y|\xi|}}{\left(1+|\xi|^{2}\right)^{(n-2) / 2}} d \xi=y^{2} \omega_{n} \int_{0}^{\infty} \frac{e^{-2 y \rho} \rho^{n-1}}{\left(1+\rho^{2}\right)^{(n-2) / 2}} d \rho \\
& =\omega_{n} \int_{0}^{\infty} \frac{e^{-2 r} r^{n-1}}{\left(y^{2}+r^{2}\right)^{(n-2) / 2}} d r
\end{aligned}
$$


где $\omega_{n}=2 \pi^{n / 2} / \Gamma(n / 2)$, то для всех $y \in(0,1]$

$$
\|u(\cdot, y)\|_{L_{2}\left(\mathbb{R}_{n}\right)}^{2} \leqslant \omega_{n} \int_{0}^{\infty} \frac{e^{-2 r} r^{n-1}}{r^{n-2}} d r=\omega_{n} / 4
$$

Покажем, что тем не менее $L_{2}$-предела при $y \rightarrow+0$ функция $u(x, y)$ не имеет. Прежде всего отметим, что если бы такой предел существовал, то он был бы равен нулю. Действительно, для любой функции $\varphi(x)$ из пространства Шварца $S(\widetilde{\varphi}(\xi)-$ преобразование Фурье этой функции, $\widetilde{\varphi}(\xi) \in S)$ имеем

$$
(u(\cdot, y), \varphi(\cdot))_{L_{2}\left(\mathbb{R}_{n}\right)}=y \int_{\mathbb{R}_{n}} \frac{e^{-y|\xi|} \overline{\widetilde{\varphi}(\xi)}}{\left(1+|\xi|^{2}\right)^{(n-2) / 4}} d \xi
$$

поэтому

$$
|(u(\cdot, y), \varphi(\cdot))| \leqslant y \int_{\mathbb{R}_{n}} \frac{|\widetilde{\varphi}(\xi)|}{\left(1+|\xi|^{2}\right)^{(n-2) / 4}} d \xi=y \cdot \text { const }
$$

и тем самњм $(u(\cdot, y), \varphi(\cdot))_{L_{2}\left(\mathbb{R}_{n}\right)} \rightarrow 0$ при $y \rightarrow+0$. Так как $S$ всюду плотно в $L_{2}\left(\mathbb{R}_{n}\right)$, а множество $\{u(\cdot, y), y>0\} \quad L_{2}\left(\mathbb{R}_{n}\right)$-ограничено, то $u(\cdot, y) \rightarrow 0$ при $y \rightarrow+0$ слабо в $L_{2}\left(\mathbb{R}_{n}\right)$, а следовательно, и сильный в $L_{2}\left(\mathbb{R}_{n}\right)$ предел функции $u(\cdot, y)$ равен нулю. Но этого быть не может, так как согласно (5)

$$
\lim _{y \rightarrow+0}\|u(\cdot, y)\|_{L_{2}\left(\mathbb{R}_{n}\right)}^{2}=\omega_{n} \int_{0}^{\infty} r e^{-2 r} d r=\omega_{n} / 4 \neq 0
$$

Обратимся теперь к условию Литлвуда-Пэли (3). В работе [4] доказано, что вьполнения условия (3) достаточно (другое достаточное условие можно получить с помощью результатов работы [3]) для существования $L_{2}$-предела на границе у решения уравнения (4) при любом $m \geqslant 1$. Однако это условие не является для этого необходимым. Функция

$$
u(x, y)=\frac{y}{(2 \pi)^{n / 2}} \int_{\mathbb{R}_{n}} e^{i(x, \xi)} \frac{e^{-y|\xi|} d \xi}{\left(1+|\xi|^{2}\right)^{(n-2) / 4}\left(\ln \left(2+|\xi|^{2}\right)\right)^{1 / 2}}, \quad(x, y) \in \Pi
$$

является бигармонической функцией (и решением уравнения (4) при любом $m \geqslant 2)$. Для всех $y \in(0,1]$ имеем неравенство

$$
\begin{aligned}
\|u(\cdot, y)\|_{L_{2}\left(\mathbb{R}_{n}\right)}^{2} & =y^{2} \int_{\mathbb{R}_{n}} \frac{e^{-2 y|\xi|} d \xi}{\left(1+|\xi|^{2}\right)^{(n-2) / 2}\left(\ln \left(2+|\xi|^{2}\right)\right)} \\
& =y^{2} \omega_{n} \int_{0}^{\infty} \frac{\rho^{n-1} e^{-2 y \rho} d \rho}{\left(1+\rho^{2}\right)^{(n-2) / 2} \ln \left(2+\rho^{2}\right)} \\
& =\omega_{n} \int_{0}^{\infty} \frac{r^{n-1} e^{-2 r} d r}{\left(y^{2}+r^{2}\right)^{(n-2) / 2} \ln \left(2+\frac{r^{2}}{y^{2}}\right)} \leqslant \omega_{n} \int_{0}^{\infty} \frac{r e^{-2 r} d r}{\ln \left(2+\frac{r^{2}}{y^{2}}\right)}
\end{aligned}
$$

из которого (согласно теореме Лебега) вытекает, что

$$
\|u(\cdot, y)\|_{L_{2}\left(\mathbb{R}_{n}\right)} \rightarrow 0 \text { при } y \rightarrow+0
$$


Таким образом, рассматриваемая функция имеет (нулевой) $L_{2}$-предел при $y \rightarrow+0$. Покажем, что условие (3) для функции $u(x, y)$ не выполняется. Действительно, легко видеть, что

$$
\begin{aligned}
\int_{\mathbb{R}_{n}}|\nabla u(x, y)|^{2} d x & =2 y^{2} \int_{\mathbb{R}_{n}} \frac{|\xi|^{2} e^{-2|\xi| y} d \xi}{\left(1+|\xi|^{2}\right)^{(n-2) / 2} \ln \left(2+|\xi|^{2}\right)} \\
& =2 \omega_{n} y^{2} \int_{0}^{\infty} \frac{r^{n+1} e^{-2 r y} d r}{\left(1+r^{2}\right)^{(n-2) / 2} \ln \left(2+r^{2}\right)} .
\end{aligned}
$$

Следовательно,

$$
\begin{aligned}
\int_{\Pi} y|\nabla u(x, y)|^{2} d x d y= & 2 \omega_{n} \int_{0}^{1} y^{3} \int_{0}^{\infty} \frac{r^{n+1} e^{-2 r y} d r}{\left(1+r^{2}\right)^{(n-2) / 2} \ln \left(2+r^{2}\right)} d y \\
= & 2 \omega_{n}\left\{\int_{1}^{\infty} \frac{r^{n+1}}{\left(1+r^{2}\right)^{(n-2) / 2} \ln \left(2+r^{2}\right)} \int_{0}^{1} y^{3} e^{-2 r y} d y d r\right. \\
& \left.+\int_{0}^{1} y^{3} \int_{0}^{1} \frac{r^{n+1} e^{-2 r y} d r}{\left(1+r^{2}\right)^{(n-2) / 2} \ln \left(2+r^{2}\right)} d y\right\} \\
= & 2 \omega_{n}\left\{\frac{3}{8} \int_{1}^{\infty} \frac{r^{n-3} d r}{\left(1+r^{2}\right)^{(n-2) / 2} \ln \left(2+r^{2}\right)}\right. \\
& +\int_{0}^{1} y^{3} \int_{0}^{1} \frac{r^{n+1} e^{-2 r y} d r}{\left(1+r^{2}\right)^{(n-2) / 2} \ln \left(2+r^{2}\right)} d y \\
& \left.-\int_{1}^{\infty} \frac{e^{-2 r}\left(\frac{1}{2 r}+\frac{3}{4 r^{2}}+\frac{3}{4 r^{3}}+\frac{3}{8 r^{4}}\right)}{\left(1+r^{2}\right)^{(n-2) / 2} \ln \left(2+r^{2}\right)} d r\right\}=+\infty
\end{aligned}
$$

поскольку первый интеграл в фигурных скобках равен $+\infty$, а два остальных сходящиеся.

Для формулировки результатов работы нам потребуются некоторые обозначения. Обозначим через $P_{N}$ при произвольном $N>0$ проекционньй оператор в $L_{2}\left(\mathbb{R}_{n}\right)$, действующий по следующему правилу: для любой функции $f(x) \in L_{2}\left(\mathbb{R}_{n}\right)$ функция $\left(P_{N} f\right)(x)$ определяется формулой

$$
\left(P_{N} f\right)(x)=\frac{1}{(2 \pi)^{n / 2}} \int_{N<|\xi|<2 N} \tilde{f}(\xi) e^{i(x, \xi)} d \xi, \quad x \in \mathbb{R}_{n}
$$

$P_{N}=F^{-1} \zeta_{N, 2 N} F$, где $F$ - оператор преобразования Фурье

$$
(F f)(\xi)=\widetilde{f}(\xi)=\frac{1}{(2 \pi)^{n / 2}} \int_{\mathbb{R}_{n}} e^{-i(x, \xi)} f(x) d x, \quad \xi \in \mathbb{R}_{n}
$$

а $\zeta_{N, 2 N}$ - оператор умножения на характеристическую функцию шарового слоя $\{N<|\xi|<2 N\}$.

Как обычно, через $W_{2}^{-1}\left(\mathbb{R}_{n}\right)$ будем обозначать гильбертово пространство, полученное пополнением пространства $L_{2}\left(\mathbb{R}_{n}\right)$ по норме

$$
\|f\|_{W_{2}^{-1}\left(\mathbb{R}_{n}\right)}=\left(\int_{\mathbb{R}_{n}} \frac{|\widetilde{f}(\xi)|^{2}}{1+|\xi|^{2}} d \xi\right)^{1 / 2} .
$$


Пусть $h=\left(h_{1}, \ldots, h_{n}\right)$ - произвольный ненулевой вектор из $\mathbb{R}_{n},|h| \neq 0$; для любой функции $f(x) \in L_{2}\left(\mathbb{R}_{n}\right)$ определим принадлежащую $L_{2}\left(\mathbb{R}_{n}\right)$ функцию

$$
\left(T_{h} f\right)(x)=\frac{\delta_{h}^{2} f(x)}{2|h|}=\frac{f(x+h)-2 f(x)+f(x-h)}{2|h|} .
$$

Равенством (7) определяется оператор из $L_{2}\left(\mathbb{R}_{n}\right)$ в $L_{2}\left(\mathbb{R}_{n}\right)$, очевидно, ограниченный при любом $h,|h| \neq 0$, однако, норма его растет при $|h| \rightarrow 0$. Под $T_{h}$ в дальнейшем будем понимать заданный формулой $(7)$ оператор, действующий из $L_{2}\left(\mathbb{R}_{n}\right)$ в $W_{2}^{-1}\left(\mathbb{R}_{n}\right)$. Покажем, что для так понимаемого оператора

$$
\left\|T_{h}\right\| \leqslant 1 \text { для всех } h \in \mathbb{R}_{n} \backslash\{0\} .
$$

Действительно, поскольку для любой функции $f(x) \in L_{2}\left(\mathbb{R}_{n}\right)$

$$
\begin{aligned}
\left(T_{h} f\right)(x)=\frac{1}{(2 \pi)^{n / 2}} & \int_{\mathbb{R}_{n}} \tilde{f}(\xi) \frac{e^{i(h, \xi)}-2+e^{-(h, \xi) i}}{2|h|} e^{i(x, \xi)} d \xi \\
\left\|T_{h} f\right\|_{W_{2}^{-1}\left(\mathbb{R}_{n}\right)}^{2} & =4 \int_{\mathbb{R}_{n}} \frac{|\tilde{f}(\xi)|^{2}}{\left(1+|\xi|^{2}\right)|h|^{2}} \sin ^{4} \frac{(\xi, h)}{2} d \xi \\
& \leqslant \int_{\mathbb{R}_{n}}|\tilde{f}(\xi)|^{2} \sin ^{2} \frac{(\xi, h)}{2} d \xi
\end{aligned}
$$

и, следовательно,

$$
\left\|T_{h} f\right\|_{W_{2}^{-1}\left(\mathbb{R}_{n}\right)}^{2} \leqslant\|f\|_{L_{2}\left(\mathbb{R}_{n}\right)}^{2},
$$

откуда вытекает неравенство (8).

Целью настоящей работы является доказательство следующих утверждений.

ТЕОРемА 1. Для того чтобъ рассматриваемое решение уравнения (1) имело $L_{2}$-предел при $y \rightarrow+0$, необходимо и достаточно, чтобь это решение было $L_{2}$-ограниченным и выполнялось условие

$$
\sup _{0<y<1}\left\|P_{N} u(\cdot, y)\right\|_{L_{2}\left(\mathbb{R}_{n}\right)} \rightarrow 0 \quad \text { npu } \quad N \rightarrow \infty .
$$

ТЕОрема 2. Для того чтобы рассматриваемое решение и $(x, y)$ уравнения (1) имело $L_{2}$-предел при $y \rightarrow+0$, необходимо и достаточно, чтобь оно бъло $L_{2}$-ограниченным и выполнялось условие

$$
\sup _{0<y<1}\left\|T_{h} u(\cdot, y)\right\|_{W_{2}^{-1}\left(\mathbb{R}_{n}\right)} \rightarrow 0 \quad \text { npu } \quad|h| \rightarrow 0 .
$$

Условие $(11)$, в котором $n$-мерная точка $h \rightarrow 0$, можно эквивалентно заменить на $n$ одномерных условий. Пусть $i_{1}=(1, \ldots, 0), \ldots, i_{n}=(0, \ldots, 0,1)$, а $h=$ $h_{1} i_{1}+\cdots+h_{n} i_{n}-$ разложение вектора $h \in \mathbb{R}_{n}$ по этому базису; тогда вместо условия (11) можно записать $n$ условий

$$
\sup _{0<y<1}\left\|T_{h_{k} i_{k}} u(\cdot, y)\right\|_{W_{2}^{-1}\left(\mathbb{R}_{n}\right)} \rightarrow 0 \quad \text { при } h_{k} \rightarrow 0, \quad k=1, \ldots, n .
$$

Эквивалентность условий (11) и $\left(11^{\prime}\right)$ доказывается в лемме 12.

Таким образом, наряду с теоремой 2 имеет место и теорема $2^{\prime}$. 
Теорема $2^{\prime}$. Для того чтобы решение $и(x, y)$ уравнения (1) имело $L_{2}$-предел при у $\rightarrow+0$, необходимо и достаточно, чтобъ оно бъло $L_{2}$-ограниченным и выполнялось условие $\left(11^{\prime}\right)$.

Обозначим через $\widetilde{u}(\xi, y), \xi \in \mathbb{R}_{n}, y \in(0,1]$, преобразование Фурье по переменным $x$ решения $u(x, y)$ уравнения $(1)$ :

$$
\widetilde{u}(\xi, y)=(F u(\cdot, y))(\xi), \quad \xi \in \mathbb{R}_{n}, \quad y \in(0,1] .
$$

Для почти всех $\xi \in \mathbb{R}_{n}$ функция $\widetilde{u}(\xi, y)$ принадлежит $W_{2}^{2 m}(a<y<1)$ при любом $a \in(0,1)$ и является решением линейного однородного дифференциального уравнения

$$
\mathscr{P}\left(\frac{d^{2}}{d y^{2}}-|\xi|^{2}\right) \widetilde{u}(\xi, y)=0, \quad y \in(0,1) .
$$

Пусть комплексные числа $z_{1}, \ldots, z_{k}, z_{i} \neq z_{j}, i \neq j, i, j=1, \ldots, k,-$ корни многочлена $\mathscr{P}(z)$, а $m_{1}, \ldots, m_{k}$ - их кратности, $m_{i} \geqslant 1, i=1, \ldots, k, m_{1}+\cdots+m_{k}=m$,

$$
\mathscr{P}(z)=\left(z-z_{1}\right)^{m_{1}} \cdots\left(z-z_{k}\right)^{m_{k}} .
$$

Тогда дифференциальное уравнение для функции $\widetilde{u}(\xi, y)$ имеет вид

$$
\prod_{j=1}^{k}\left(\frac{d^{2}}{d y^{2}}-\left(|\xi|^{2}+z_{j}\right)\right)^{m_{j}} \widetilde{u}(\xi, y)=0, \quad 0<y<1 .
$$

При каждом $j, j=1, \ldots, k$, в комплексной $z$-плоскости проведем разрез по лучу $z=-z_{j}+t,-\infty<t \leqslant 0$; под функцией $\sqrt{z+z_{j}}, 1 \leqslant j \leqslant k$, в таким образом разрезанной плоскости будем понимать ту ветвь корня, для которой $\left.\left(\sqrt{z+z_{j}}-\sqrt{z}\right)\right|_{\operatorname{Im} z=0} \rightarrow 0$ при $\operatorname{Re} z \rightarrow+\infty$, где под $\left.\sqrt{z}\right|_{\operatorname{Im} z=0, \operatorname{Re} z>0}$ понимается положительное значение корня $\sqrt{\operatorname{Re} z}$.

Функции

$$
\lambda_{j}=\lambda_{j}(\xi)=\sqrt{|\xi|^{2}+z_{j}}, \quad j=1, \ldots, k, \quad \xi \in \mathbb{R}_{n}
$$

являются характеристическими числами обькновенного дифференциального уравнения

$$
\prod_{j=1}^{k}\left(\frac{d}{d y}-\lambda_{j}(\xi)\right)^{m_{j}} v=0, \quad y \in \mathbb{R}_{1}, \quad \xi \in \mathbb{R}_{n},
$$

и поскольку для всех $\xi \in \mathbb{R}_{n} \lambda_{j} \neq \lambda_{s}, j \neq s, j, s=1, \ldots, k$, то кратность характеристического числа $\lambda_{j}$ при всех $\xi \in \mathbb{R}_{n}$ равна $m_{j}, j=1, \ldots, k$. Числа $-\lambda_{j}$, $j=1, \ldots, k$, являются характеристическими числами уравнения

$$
\prod_{j=1}^{k}\left(\frac{d}{d y}+\lambda_{j}(\xi)\right)^{m_{j}} w=0, \quad y \in \mathbb{R}_{1}, \quad \xi \in \mathbb{R}_{n},
$$

и кратность $-\lambda_{j}$ при всех $\xi \in \mathbb{R}_{n}$ равна $m_{j}, j=1, \ldots, k$. Поэтому при всех $\xi \in \mathbb{R}_{n}$ система функций

$$
\mathrm{I}=\left(e^{\lambda_{1} y}, \ldots, y^{m_{1}-1} e^{\lambda_{1} y}, \ldots, e^{\lambda_{k} y}, \ldots, y^{m_{k}-1} e^{\lambda_{k} y}\right)
$$


является фундаментальной системой решений уравнения (14), а система

$$
\mathrm{II}=\left(e^{-\lambda_{1} y}, \ldots, y^{m_{1}-1} e^{-\lambda_{1} y}, \ldots, e^{-\lambda_{k} y}, \ldots, y^{m_{k}-1} e^{-\lambda_{k} y}\right)
$$

является фундаментальной системой решений уравнения (15).

Объединение этих систем, т.е. система

$$
\mathrm{III}=\left(e^{\lambda_{1} y}, \ldots, y^{m_{1}-1} e^{\lambda_{1} y}, \ldots, e^{-\lambda_{k} y}, \ldots, y^{m_{k}-1} e^{-\lambda_{k} y}\right)
$$

будет фундаментальной системой решений уравнения (12) для всех $\xi \in \mathbb{R}_{n}$, кроме тех значений $\xi$, для которых при каком-либо $j, j=1, \ldots, k$, имеет место равенство $\lambda_{j}=-\lambda_{j}$ (равенство $\lambda_{j}=-\lambda_{r}$ при $j \neq r$, очевидно, невозможно), которое в силу (13) может быть лишп при вешественном неположительном значении корня $z_{j}$ многочлена $\mathscr{P}$. Это множество значений $\xi$ совпадает со сферой $\left\{|\xi|=\sqrt{-z_{j}}\right\}$, на этой сфере общая кратность корня $\lambda_{j}=-\lambda_{j}$ равна $2 m_{j}$. Таким образом, система III является фундаментальной системой решений уравнения (12) для всех $\xi \in \mathbb{R}_{n} \backslash Z$, где $Z=\bigcup_{j=1}^{k}\left\{|\xi|=\sqrt{-z_{j}}\right\}$ (множество $Z$ пусто, если среди корней многочлена $\mathscr{P}$ нет вещественных неотрицательных чисел), или, более грубо, для всех $\xi \in \mathbb{R}_{n}$, для которых $|\xi|>R_{0}=\max \left\{\sqrt{\left|z_{1}\right|} ; \ldots ; \sqrt{\left|z_{k}\right|}\right\}$.

В дальнейших наших построениях будут использованы другие фундаментальные системы решений уравнений $(14),(15)$ и (12). Для построения нужной нам системы

$$
\widetilde{\mathrm{II}}=\left(w_{1}(\xi, y), \ldots, w_{m}(\xi, y)\right)
$$

являющейся фундаментальной системой решений уравнения (15), поступим следующим образом. Пусть $s$ - произвольное целое число из отрезка $[1, m]$; обозначим через $\varkappa=\varkappa(s), 1 \leqslant \varkappa \leqslant k$, номер того полуинтервала из $\left(0, m_{1}\right],\left(m_{1}, m_{1}+m_{2}\right]$, $\ldots,\left(m_{1}+\cdots+m_{k-1}, m\right]$ (напомним, что $m_{1}+\cdots+m_{k}=m$ ), в котором содержится $s$, и положим

$$
\begin{gathered}
w_{s}(\xi, y)=\frac{1}{2 \pi i} \int_{|z|=|\xi|+R_{0}+1} \frac{e^{z y} d z}{\left(z+\lambda_{1}\right)^{m_{1}} \cdots\left(z+\lambda_{\varkappa-1}\right)^{m_{\varkappa-1}\left(z+\lambda_{\varkappa}\right)^{s-\left(m_{1}+\cdots+m_{\varkappa-1}\right)}}} \\
1 \leqslant s \leqslant m, \quad \varkappa=\varkappa(s) \in[1, k], \quad \xi \in \mathbb{R}_{n}, \quad y \in[0,1],
\end{gathered}
$$

где интеграл берется по окружности с центром в начале координат радиуса $|\xi|+R_{0}+1$ комплексной $z$-плоскости. В силу неравенств $\left|\lambda_{p}\right| \leqslant \sqrt{|\xi|^{2}+\left|z_{p}\right|} \leqslant$ $|\xi|+\sqrt{\left|z_{p}\right|}, p=1, \ldots, k$, все полюсы подынтегральной функции в (16) содержатся в круге $\left\{|z|<|\xi|+R_{0}+1\right\}$. Очевидно, что функция $w_{s}(\xi, y)$ при всех $s=1, \ldots, m$ и всех $\xi \in \mathbb{R}_{n}$ является решением уравнения (15).

Вычисляя интеграл в (16) с помощью вьгетов, получим: для $s \leqslant m_{1}$

$$
w_{s}(\xi, y)=\frac{1}{(s-1) !} y^{s-1} e^{-\lambda_{1} y}, \quad \xi \in \mathbb{R}_{n}, \quad y \in[0,1]
$$


а для $s>m_{1}$

$$
\begin{aligned}
w_{s}(\xi, y)= & \left.\frac{1}{\left(m_{1}-1\right) !} \frac{\partial^{m_{1}-1}}{\partial z^{m_{1}-1}}\left(\frac{e^{z y}}{\left(z+\lambda_{2}\right)^{m_{2}} \cdots\left(z+\lambda_{\varkappa}\right)^{s-\left(m_{1}+\cdots+m_{\varkappa-1}\right)}}\right)\right|_{z=-\lambda_{1}} \\
& +\cdots+\frac{1}{\left(s-\left(m_{1}+\cdots+m_{\varkappa-1}\right)-1\right) !} \frac{\partial^{s-\left(m_{1}+\cdots+m_{\varkappa-1}\right)-1}}{\partial z^{s-\left(m_{1}+\cdots+m_{\varkappa-1}\right)-1}} \\
& \times\left.\left(\frac{e^{z y}}{\left(z+\lambda_{1}\right)^{m_{1}} \cdots\left(z+\lambda_{\varkappa-1}\right)^{m_{\varkappa-1}}}\right)\right|_{z=-\lambda_{\varkappa}} .
\end{aligned}
$$

Следовательно, матрица $C=C(\xi)$, устанавливающая следуюшую связь между фундаментальной системой II и системой II

$$
\widetilde{\mathrm{II}}^{*}=C \mathrm{II}^{*}
$$

(векторы II* $^{*} \widetilde{\mathrm{II}}^{*}$ - соответствующие векторы-столбцы для векторов-строк II и II), является треугольной, причем $s$-й элемент диагонали матрицы $C$ при $s \leqslant m_{1}$ равен $1 /(s-1)$ !, а при $s>m_{1}$ равен

$$
\frac{1}{\left(s-\left(m_{1}+\cdots+m_{\varkappa-1}\right)-1\right) !\left(\lambda_{1}-\lambda_{\varkappa}\right)^{m_{1}} \cdots\left(\lambda_{\varkappa-1}-\lambda_{\varkappa}\right)^{m_{\varkappa-1}}} \neq 0 .
$$

Это означает, что система $\widetilde{\mathrm{II}}=\left\{w_{1}(\xi, y), \ldots, w_{m}(\xi, y)\right\}$, как и система II, является фундаментальной системой решений уравнения (15) при всех $\xi \in \mathbb{R}_{n}$.

Аналогично, система

$$
\left\{v_{1}(\xi, y), \ldots, v_{m}(\xi, y)\right\}
$$

где

$$
\begin{gathered}
v_{m}(\xi, y)=\frac{1}{2 \pi i} \int_{|z|=|\xi|+R_{0}+1} \frac{e^{-z y}}{\left(z+\lambda_{1}\right)^{m_{1}} \cdots\left(z-\lambda_{\varkappa}\right)^{s-\left(m_{1}+\cdots+m_{\varkappa-1}\right)}} d z \\
s=1, \ldots, m, \quad \xi \in \mathbb{R}_{n}, \quad y \in[0,1]
\end{gathered}
$$

является при всех $\xi \in \mathbb{R}_{n}$ фундаментальной системой решений уравнения (14), а система

$$
\left\{v_{1}(\xi, y), \ldots, v_{m}(\xi, y), w_{1}(\xi, y), \ldots, w_{m}(\xi, y)\right\}
$$

- объединение систем $\left\{v_{s}(\xi, y), s=1, \ldots, m\right\}$ и $\left\{w_{s}(\xi, y), s=1, \ldots, m\right\}$ - является фундаментальной системой решений уравнения (12) при всех $\xi \in \mathbb{R}_{n} \backslash Z$ или, более грубо, для всех $\xi \in \mathbb{R}_{n}$, для которых $|\xi|>R_{0}$. Это означает, что для функции $\widetilde{u}(\xi, y)$ - преобразования Фурье исследуемого решения - однозначно найдутся при $|\xi|>R_{0}$ такие функции $A_{1}(\xi), \ldots, A_{m}(\xi), B_{1}(\xi), \ldots, B_{m}(\xi)$, что

$$
\widetilde{u}(\xi, y)=\sum_{i=1}^{m} B_{i}(\xi) v_{i}(\xi, y)+\sum_{i=1}^{m} A_{i}(\xi) w_{i}(\xi, y), \quad y \in(0,1), \quad|\xi|>R_{0}+1
$$

Следующие леммы посвящены изучению свойств функций $v_{i}(\xi, y), w_{i}(\xi, y)$, $i=1, \ldots, m$, и коэффициентов $B_{i}(\xi)$ и $A_{i}(\xi), i=1, \ldots, m$, в представлении $(17)$. 
Лемма 1. Для всех $\xi \in \mathbb{R}_{n}$ функции $w_{s}(\xi, y), y \in[0,1]$, удовлетворяют условиям

$$
\begin{gathered}
\left.w_{1}\right|_{y=0}=1, \\
\left.w_{s}\right|_{y=0}=\cdots=\left.\frac{\partial^{s-2} w_{s}}{\partial y^{s-2}}\right|_{y=0}=0,\left.\quad \frac{\partial^{s-1} w_{s}}{\partial y^{s-1}}\right|_{y=0}=1, \quad s=2, \ldots, m .
\end{gathered}
$$

При каждом $s, 1 \leqslant s \leqslant m$, равномерно по $y \in[0,1]$

$$
w_{s}(\xi, y)=\frac{e^{-y|\xi|} y^{s-1}}{(s-1) !}\left(1+O\left(\frac{1}{|\xi|}\right)\right) \quad \text { npu } \quad|\xi| \rightarrow \infty .
$$

Существует постоянная $R_{1}, R_{1} \geqslant R_{0}+1$, такая, что для каждого $s$, $1 \leqslant s \leqslant m$,

$$
\frac{e^{-y|\xi|} y^{s-1}}{2(s-1) !} \leqslant\left|w_{s}(\xi, y)\right| \leqslant 2 \frac{e^{-y|\xi|} y^{s-1}}{(s-1) !}
$$

для всех $y \in[0,1],|\xi| \geqslant R_{1}$.

Соответствующие свойства функций $v_{s}(\xi, y), s=1, \ldots, m$, устанавливаются в лемме $1^{\prime}$.

Лемма $1^{\prime}$. Для всех $\xi \in \mathbb{R}_{n}$ функиии $v_{s}(\xi, y), y \in[0,1], 1 \leqslant s \leqslant m$, удовлетворяют условиям

$$
\begin{gathered}
\left.v_{1}\right|_{y=0}=1 \\
\left.v_{s}\right|_{y=0}=\cdots=\left.\frac{\partial^{s-2} v_{s}}{\partial y^{s-2}}\right|_{y=0}=0,\left.\quad \frac{\partial^{s-1} v_{s}}{\partial y^{s-1}}\right|_{y=0}=1, \quad s=2, \ldots, m .
\end{gathered}
$$

При каждом $s, 1 \leqslant s \leqslant m$, равномерно по $y \in[0,1]$

$$
v_{s}(\xi, y)=\frac{e^{y|\xi|} y^{s-1}}{(s-1) !}\left(1+O\left(\frac{1}{|\xi|}\right)\right) \quad \text { npu } \quad|\xi| \rightarrow \infty .
$$

Существует постоянная $R_{1}, R_{1} \geqslant R_{0}+1$, такая, что для каждого $s$, $1 \leqslant s \leqslant m$,

$$
\frac{e^{y|\xi|} y^{s-1}}{2(s-1) !} \leqslant\left|v_{s}(\xi, y)\right| \leqslant 2 \frac{e^{y|\xi|} y^{s-1}}{(s-1) !} \quad \text { для } \quad \text { всех } \quad y \in[0,1], \quad|\xi| \geqslant R_{1} .
$$

Леммы 1 и $1^{\prime}$ доказываются совершенно одинаково, поэтому остановимся на доказательстве лишь леммы 1.

ДоКАЗАТЕЛЬСТво ЛЕммы 1 . Для вычисления значений функций $w_{s}(\xi, y)$ и их производных по $y$ при $y=0$ следует воспользоваться очевидными равенствами

$$
\begin{aligned}
\frac{1}{2 \pi i} \int_{|z|=|\xi|+R_{0}+1} \frac{z^{p} d z}{\left(z+\lambda_{1}\right)^{m_{1}} \cdots\left(z+\lambda_{\varkappa}\right)^{s-\left(m_{1}+\cdots+m_{\varkappa-1}\right)}} \\
= \begin{cases}0 & \text { при } 0 \leqslant p \leqslant s-2, \\
1 & \text { при } p=s-1,\end{cases}
\end{aligned}
$$

из которых немедленно вытекают требуемые соотношения. 
Для доказательства асимптотического равенства (18) воспользуемся разложением

$$
\lambda_{j}=\lambda_{j}(\xi)=|\xi| \sqrt{1+\frac{z_{j}}{|\xi|^{2}}}=|\xi|+\frac{z_{j}}{2|\xi|}+\sum_{s=1}^{\infty} \frac{(-1)^{s}(2 s-1) ! !}{2^{s+1}(s+1) !} \frac{z_{j}^{s+1}}{|\xi|^{2 s+1}}
$$

в котором ряд сходится при $|\xi|>\sqrt{\left|z_{j}\right|}, j=1, \ldots, k$. Соотношения (18) при $s \leqslant m_{1}$, при которых, как уже отмечалось, $w_{s}=e^{-\lambda_{1}(\xi) y} y^{s-1} /(s-1)$ !, являются прямыми следствиями (21). Пусть $s>m_{1}$. Под знаком интеграла в (16) сделаем замену переменной $z=\zeta|\xi|$ (считая, что $|\xi| \geqslant 1$ ), а затем, обозначив $\zeta$ через $z-1$, получим

$$
\begin{aligned}
& w_{s}(\xi, y)=\frac{1}{2 \pi i|\xi|^{s-1}} \int_{|\zeta|=1+\frac{R_{0}+1}{|\xi|}} \frac{e^{y|\xi| \zeta} d \zeta}{\left(\zeta+\frac{\lambda_{1}}{|\xi|}\right)^{m_{1}} \cdots\left(z+\frac{\lambda_{\varkappa}}{|\xi|}\right)^{s-\left(m_{1}+\cdots+m_{\varkappa-1}\right)}} \\
& =\frac{e^{-y|\xi|}}{2 \pi i|\xi|^{s-1}} \int_{|z-1|=1+\frac{R_{0}+1}{|\xi|}} \frac{e^{y|\xi| z} d z}{\left(z-\left(1-\frac{\lambda_{1}}{|\xi|}\right)\right)^{m_{1}} \cdots\left(z-\left(1-\frac{\lambda_{\varkappa}}{|\xi|}\right)\right)^{s-\left(m_{1}+\cdots+m_{\varkappa-1}\right)}} \\
& =\frac{e^{-y|\xi|}}{2 \pi i|\xi|^{s-1}} \int_{|z|=1} \frac{e^{y|\xi| z} d z}{\left(z-\left(1-\frac{\lambda_{1}}{|\xi|}\right)\right)^{m_{1}} \cdots\left(z-\left(1-\frac{\lambda_{\varkappa}}{|\xi|}\right)\right)^{s-\left(m_{1}+\cdots+m_{\varkappa-1}\right)}},
\end{aligned}
$$

при этом считаем $|\xi|$ настолько большим, что все полюсы подынтегральной функции находятся в круге $\{|z|<1\}$ : из (21) вытекает, что при любом $p, p=1, \ldots, k$, $\frac{\lambda_{p}}{|\xi|}-1=\frac{z_{p}}{2|\xi|^{2}}+o\left(\frac{1}{|\xi|^{2}}\right)$. Поскольку для всех (комплексных) $z$ и любого целого $r \geqslant 0$

$$
e^{z}-\left(1+\frac{z}{1 !}+\cdots+\frac{z^{r}}{r !}\right)=\frac{1}{r !} \int_{0}^{z}(z-\theta)^{r} e^{\theta} d \theta
$$

где интегрирование производится по отрезку, соединяющему точки 0 и $z$, то в силу равенств (20) имеем

$$
\begin{aligned}
& w_{s}(\xi, y)=\frac{e^{-y|\xi|}}{2 \pi i|\xi|^{s-1}} \\
& \times \int_{|z|=1} \frac{\left(\frac{1}{(s-1) !} \int_{0}^{y|\xi| z}(y|\xi| z-\theta)^{s-1} e^{\theta} d \theta+1+\frac{y|\xi| z}{1 !}+\cdots+\frac{(y|\xi| z)^{s-1}}{(s-1) !}\right) d z}{\left(z-\left(1-\frac{\lambda_{1}}{|\xi|}\right)\right)^{m_{1}} \cdots\left(z-\left(1-\frac{\lambda_{\varkappa}}{|\xi|}\right)\right)^{s-\left(m_{1}+\cdots+m_{\varkappa-1}\right)}} \\
& =\frac{e^{-y|\xi|}}{2 \pi i(s-1) !|\xi|^{s-1}} \\
& \times\left\{\int_{|z|=1} \frac{\int_{0}^{y|\xi| z}(y|\xi| z-\theta)^{s-1} e^{\theta} d \theta d z}{\left(z-\left(1-\frac{\lambda_{1}}{|\xi|}\right)\right)^{m_{1}} \cdots\left(z-\left(1-\frac{\lambda_{\varkappa}}{|\xi|}\right)\right)^{s-\left(m_{1}+\cdots+m_{\varkappa-1}\right)}+2 \pi i(y|\xi|)^{s-1}}\right\} \\
& \left.=\frac{e^{-y|\xi|}}{2 \pi i(s-1) !|\xi|^{s-1}} \int_{|z|=1} \frac{(y|\xi| z)^{s} \int_{0}^{1}(1-t)^{s-1} e^{y|\xi| z t} d t d z}{\left(z-\left(1-\frac{\lambda_{1}}{|\xi|}\right)\right)^{m_{1}} \cdots\left(z-\left(1-\frac{\lambda_{\varkappa}}{|\xi|}\right)\right)^{s-\left(m_{1}+\cdots+m_{\varkappa-1}\right)}}\right\} \\
& \times\left\{2 \pi i(y|\xi|)^{s-1}+\int^{s}(z)\right.
\end{aligned}
$$




$$
\begin{aligned}
& =\frac{e^{-y|\xi|} y^{s-1}}{(s-1) !} \\
& \times\left\{1+\frac{y|\xi|}{2 \pi i} \int_{|z|=1} \frac{z^{s} \int_{0}^{1}(1-t)^{s-1} e^{y|\xi| z t} d t d z}{\left(z-\left(1-\frac{\lambda_{1}}{|\xi|}\right)\right)^{m_{1}} \cdots\left(z-\left(1-\frac{\lambda_{\varkappa}}{|\xi|}\right)\right)^{s-\left(m_{1}+\cdots+m_{\varkappa-1}\right)}}\right\} .
\end{aligned}
$$

Поэтому для доказательства (18) достаточно проверить, что равномерно по $y \in[0,1]$ и $t \in[0,1]$ при $|\xi| \rightarrow \infty$

$$
\frac{1}{2 \pi i} \int_{|z|=1} \frac{z^{s} e^{y t|\xi| z} d z}{\left(z-\mu_{1}\right)^{m_{1}} \cdots\left(z-\mu_{\varkappa}\right)^{s-\left(m_{1}+\cdots+m_{\varkappa-1}\right)}}=O\left(\frac{1}{|\xi|^{2}}\right)
$$

где $\mu_{i}=\mu_{i}(\xi)=1-\lambda_{i}(\xi) /|\xi|, i=1, \ldots, k$. В силу $(21)$ при $|\xi| \rightarrow \infty$ имеют место соотношения $\mu_{i}=O\left(1 /|\xi|^{2}\right)$ и $\mu_{i}-\mu_{j}=\frac{z_{i}-z_{j}}{|\xi|^{2}}+O\left(1 /|\xi|^{3}\right), z_{i} \neq z_{j}, i \neq j$, $i, j=1, \ldots, k$.

Для доказательства (22) вычислим интеграл в (22) с помощью вычетов и проверим, что соотношению (22) удовлетворяет вычет подынтегральной функции в каждом из ее полюсов. Вычет в полюсе $\mu_{1}$ имеет вид

$$
\begin{aligned}
\operatorname{Res}_{z=\mu_{1}} & \frac{z^{s} e^{y t|\xi| z}}{\left(z-\mu_{1}\right)^{m_{1}} \cdots\left(z-\mu_{\varkappa}\right)^{s-\left(m_{1}+\cdots+m_{\varkappa-1}\right)}} \\
\quad= & \left.\frac{1}{\left(m_{1}-1\right) !} \frac{d^{m_{1}-1}}{d z^{m_{1}-1}}\left(\frac{z^{y t|\xi| z}}{\left(z-\mu_{2}\right)^{m_{1}} \cdots\left(z-\mu_{\varkappa}\right)^{s-\left(m_{1}+\cdots+m_{\varkappa-1}\right)}}\right)\right|_{z=\mu_{1}} \\
\quad= & \left.\sum_{p+q+r=m_{1}-1} C_{p q r}\left(\frac{d^{p} e^{y t|\xi| z}}{d z^{p}}\right)\left(\frac{d^{q} z^{s}}{d z^{q}}\right)\left(\frac{d^{r}}{d z^{r}}\left(\frac{1}{\left(z-\mu_{2}\right)^{m_{1}} \cdots}\right)\right)\right|_{z=\mu_{1}},
\end{aligned}
$$

где $C_{p q r}$ - соответствуюшие биномиальные коэффициенты. В силу (21) равномерно по $y \in[0,1]$ и $t \in[0,1]$

$$
\left.\frac{d^{p} e^{y t|\xi| z}}{d z^{p}}\right|_{z=\mu_{1}}=y^{p} t^{p}|\xi|^{p} e^{y t|\xi| \mu_{1}}=O\left(|\xi|^{p}\right) \quad \text { при }|\xi| \rightarrow \infty
$$

а также

$$
\left.\frac{d^{q} z^{s}}{d z^{q}}\right|_{z=\mu_{1}}=O\left(\frac{1}{|\xi|^{2(s-q)}}\right) \text { при }|\xi| \rightarrow \infty
$$

Далее, $\frac{\partial^{r}}{\partial z^{r}} \frac{1}{\left(z-\mu_{2}\right)^{m_{2}} \cdots\left(z-\mu_{\varkappa}\right)^{s-\left(m_{1}+\cdots+m_{\varkappa-1}\right)}}$ есть линейная комбинация нескольких дробей вида $\frac{1}{\left(z-\mu_{2}\right)^{\alpha_{2}} \cdots\left(z-\mu_{\varkappa}\right)^{\alpha}}$, степени многочленов в знаменателях которых на $r$ единиц больше степени многочлена в знаменателе дроби, которая дифференцируется, т.е.

$$
\alpha_{2}+\cdots+\alpha_{\varkappa}=r+m_{2}+\cdots+m_{\varkappa-1}+s-\left(m_{1}+\cdots+m_{\varkappa-1}\right)=s-m_{1}+r .
$$


Ho

$$
\begin{aligned}
& \left.\frac{1}{\left(z-\mu_{2}\right)^{\alpha_{2}} \cdots\left(z-\mu_{\varkappa}\right)^{\alpha_{\varkappa}}}\right|_{z=\mu_{1}}=\frac{1}{\left(\mu_{1}-\mu_{2}\right)^{\alpha_{2}} \cdots\left(\mu_{1}-\mu_{\varkappa}\right)^{\alpha_{\varkappa}}} \\
& =\frac{|\xi|^{2\left(\alpha_{2}+\cdots+\alpha_{\varkappa}\right)}}{\left(z_{2}-z_{1}\right)^{\alpha_{2} \cdots\left(z_{\varkappa}-z_{1}\right)^{\alpha_{\varkappa}}}}=O\left(|\xi|^{2\left(s-m_{1}+r\right)}\right) \text { при }|\xi| \rightarrow \infty,
\end{aligned}
$$

поэтому

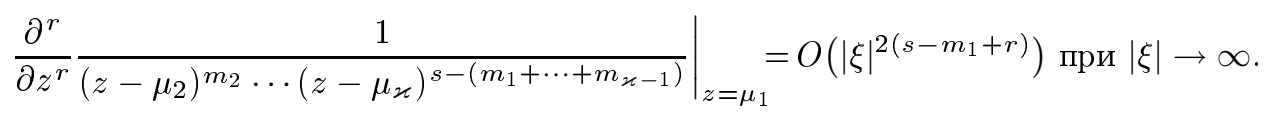

Таким образом, равномерно по $y \in[0,1]$ и $t \in[0,1]$ при $|\xi| \rightarrow \infty$

$$
\begin{aligned}
& \operatorname{Res}_{z=\mu_{1}} \frac{z^{s} e^{y t|\xi| z}}{\left(z-\mu_{1}\right)^{m_{1}} \cdots\left(z-\mu_{\varkappa}\right)^{s-\left(m_{1}+\cdots+m_{\varkappa-1}\right)}} \\
& \quad=O\left(|\xi|^{p-2 s+2 q+2 s-2 m_{1}+2 r}\right)=O\left(|\xi|^{2(p+q+r)-p-2 m_{1}}\right) \\
& \quad=O\left(|\xi|^{-p-2}\right)=O\left(|\xi|^{-2}\right)
\end{aligned}
$$

поскольку $p \geqslant 0$. Аналогично рассматриваются и другие полюсы подынтегральной функции. Таким образом, соотношение (22) установлено, а вместе с ним установлено и равенство (18).

Неравенства (19), конечно, являются простыми следствиями равенства (18). Леммы 1 и $1^{\prime}$ доказаны.

Первоначальные свойства коэффициентов $A_{i}(\xi)$ и $B_{i}(\xi), i=1, \ldots, m$, в разложении (17) по фундаментальной системе решений преобразования Фурье исследуемого решения уравнения (1) установлены в следуюшей лемме 2 ; из нее, в частности, вытекает, что эти коэффициенты являются локально интегрируемыми функциями вне шара достаточно большого радиуса.

Лемма 2. Существует такое $R_{2}>0, R_{2} \geqslant R_{1}$, что при любом $\varepsilon \in(0,1 / 2)$ функиии

$$
A_{i}(\xi) e^{-\varepsilon|\xi|} \in L_{2}\left(|\xi|>R_{2}\right), \quad i=1, \ldots, m,
$$

и функции

$$
B_{i}(\xi) e^{(1-\varepsilon)|\xi|} \in L_{2}\left(|\xi|>R_{2}\right), \quad i=1, \ldots, m .
$$

Для доказательства этой леммы поступим следуюшим образом. Возьмем произвольные $\nu$ и $\sigma, 0<\sigma<\nu<1$, и рассмотрим $2 m$ чисел $y_{1}=\nu-\frac{\sigma}{2 m}$, $y_{2}=\nu-\frac{2 \sigma}{2 m}, \ldots, y_{k}=\nu-\frac{k \sigma}{2 m}, \ldots, y_{2 m}=\nu-\frac{2 m \sigma}{2 m}=\nu-\sigma>0$. Обозначим через $g_{p}(\xi)$ значение функции $\widetilde{u}(\xi, y)$ при $y=y_{p}$ :

$$
\widetilde{u}\left(\xi, y_{p}\right)=g_{p}(\xi), \quad p=1, \ldots, 2 m ;
$$

$g_{p}(\xi) \in L_{2}\left(\mathbb{R}_{n}\right), p=1, \ldots, 2 m$. В силу (17) получим $2 m$ равенств, представляющих собой систему $2 m$ уравнений относительно $2 m$ функций $B_{1}(\xi), \ldots, B_{m}(\xi)$, $A_{1}(\xi), \ldots, A_{m}(\xi),|\xi|>R_{0}+1$ :

$$
\sum_{j=1}^{m} B_{j}(\xi) v_{j}\left(\xi, y_{p}\right)+\sum_{j=1}^{m} A_{j}(\xi) w_{j}\left(\xi, y_{p}\right)=g_{p}(\xi),|\xi|>R_{0}+1, p=1, \ldots, 2 m
$$


Найдем оценки при больших $|\xi|$ определителя $\Delta(\xi)$ (определителя $2 m$-го порядка) этой системы и его миноров $\Delta_{p q}(\xi)(2 m-1)$-го порядка, получающихся из определителя $\Delta(\xi)$ вычеркиванием его $p$-й строки и $q$-го столбца; при этом будем пользоваться асимптотическими равенствами из лемм 1 и $1^{\prime}$. Разложим определитель $\Delta(\xi)$ согласно теореме Лапласа по первым $m$ столбцам. Среди всех миноров $m$-го порядка, расположенных в первых $m$ столбцах определителя $\Delta(\xi)$, максимальным по порядку при $|\xi| \rightarrow \infty$ является минор $\Delta^{\prime}(\xi)$, занимающий крайнее верхнее положение, и отношение любого другого минора $m$-го порядка, расположенного в этих столбцах, к $\Delta^{\prime}(\xi)$ при $|\xi| \rightarrow \infty$ есть $O\left(e^{-\frac{\sigma|\xi|}{2 m}}\right)$. Аналогично, среди всех миноров $m$-го поря дка, расположенных в последних $m$ столбцах определителя $\Delta(\xi)$, максимальным по порядку при $|\xi| \rightarrow \infty$ является минор $\Delta^{\prime \prime}(\xi)$, занимающий крайнее нижнее положение, и отношение любого другого минора $m$-го порядка, расположенного в этих столбцах, к $\Delta^{\prime \prime}(\xi)$ при $|\xi| \rightarrow \infty$ есть $O\left(e^{-\frac{\sigma|\xi|}{2 m}}\right)$. Следовательно,

$$
\Delta(\xi)=\Delta^{\prime}(\xi) \Delta^{\prime \prime}(\xi)\left(1+O\left(e^{-\frac{\sigma|\xi|}{2 m}}\right)\right) \text { при }|\xi| \rightarrow \infty .
$$

Из равенств $(18)$ и $\left(18^{\prime}\right)$ вытекает, что

$$
\begin{aligned}
\Delta^{\prime}(\xi) & =e^{|\xi|\left(y_{1}+\cdots+y_{m}\right)} \frac{W\left(y_{1}, \ldots, y_{m}\right)}{1 ! 2 ! \cdots(m-1) !}\left(1+O\left(\frac{1}{|\xi|}\right)\right) \text { при }|\xi| \rightarrow \infty, \\
\Delta^{\prime \prime}(\xi) & =e^{-|\xi|\left(y_{m+1}+\cdots+y_{2 m}\right)} \frac{W\left(y_{m+1}, \ldots, y_{2 m}\right)}{1 ! 2 ! \cdots(m-1) !}\left(1+O\left(\frac{1}{|\xi|}\right)\right) \text { при }|\xi| \rightarrow \infty,
\end{aligned}
$$

где $W\left(y_{1}, \ldots, y_{m}\right)$ - определитель Вандермонда,

$$
\begin{gathered}
W\left(y_{1}, \ldots, y_{m}\right)=\prod_{1 \leqslant i<j \leqslant m}\left(y_{i}-y_{j}\right)=\left(\frac{\sigma}{2 m}\right)^{\frac{m(m-1)}{2}} 1 ! \cdots(m-1) !, \\
W\left(y_{m+1}, \ldots, y_{2 m}\right)=W\left(y_{1}, \ldots, y_{m}\right),
\end{gathered}
$$

и тем самым

$$
\begin{aligned}
\Delta(\xi) & =e^{|\xi|\left(y_{1}+\cdots+y_{m}-y_{m+1}-\ldots-y_{2 m}\right)}\left(\frac{\sigma}{2 m}\right)^{m(m-1)}\left(1+O\left(\frac{1}{|\xi|}\right)\right) \\
& =e^{\frac{\sigma m}{2}|\xi|}\left(1+O\left(\frac{1}{|\xi|}\right)\right) \text { при }|\xi| \rightarrow \infty
\end{aligned}
$$

Следовательно, существует такое число $R_{2}, R_{2} \geqslant R_{1}$, что

$$
|\Delta(\xi)| \geqslant \frac{1}{2} e^{\frac{\sigma m}{2}|\xi|} \text { для всех } \xi,|\xi| \geqslant R_{2} .
$$

Оценим миноры $\Delta_{p q}(\xi)$. Пусть сначала $p \in[1, m]$, а $q$ - произвольное, $1 \leqslant q \leqslant 2 m$. Разлагая определитель $\Delta_{p q}(\xi)$ согласно теореме Лапласа по первьм $(m-1)$-му столбцу и пользуясь приведенными выше соображениями, получим с помощью (18) и $\left(18^{\prime}\right)$ при достаточно больших $|\xi|,|\xi| \geqslant R_{2}$, оценку

$$
\begin{aligned}
\left|\Delta_{p q}(\xi)\right| & \leqslant C_{p q} e^{|\xi|\left(y_{1}+\cdots+y_{m-1}\right)-|\xi|\left(y_{m+1}+\cdots+y_{2 m}\right)} \\
& =C_{p q} e^{|\xi|\left((m-1) \nu-\frac{\sigma}{2 m} \frac{m(m-1)}{2}\right)-|\xi|\left(m \nu-\frac{\sigma}{2 m} \frac{m(3 m+1)}{2}\right)} \\
& =C_{p q} e^{|\xi|\left(-\nu+\frac{\sigma}{2}(m+1)\right)}, \quad|\xi| \geqslant R_{2}
\end{aligned}
$$


где $C_{p q}$ - некоторые положительные постоянные. Если $p \in[m+1,2 m]$, то при любом $q, 1 \leqslant q \leqslant 2 m$, разлагая определитель $\Delta_{p q}(\xi)$ по первым $m$ столбцам, аналогично получим неравенство

$$
\begin{aligned}
\left|\Delta_{p q}(\xi)\right| & \leqslant C_{p q} e^{|\xi|\left(y_{1}+\cdots+y_{m}\right)-|\xi|\left(y_{m+2}+\cdots+y_{2 m}\right)} \\
& =C_{p q} e^{|\xi|\left(m \nu-\frac{\sigma}{2 m} \frac{m(m+1)}{2}\right)-|\xi|\left((m-1) \nu-\frac{\sigma}{2 m} \frac{(m-1)(3 m+2)}{2}\right)} \\
& =C_{p q} e^{|\xi|\left(\nu+\frac{\sigma}{2 m}\left(m^{2}-m-1\right)\right)}, \quad|\xi| \geqslant R_{2} .
\end{aligned}
$$

В результате из $(25),(26)$ и $(27)$ получаем для всех $p, 1 \leqslant p \leqslant m$, оценки

$$
\begin{aligned}
\left|B_{p}(\xi)\right| & \leqslant \sum_{q=1}^{2 m}\left|g_{q}(\xi)\right| \frac{\left|\Delta_{p q}(\xi)\right|}{|\Delta(\xi)|} \\
& \leqslant 2 e^{-|\xi|\left(\nu-\frac{\sigma}{2}\right)} \sum_{q=1}^{2 m} C_{p q}\left|g_{q}(\xi)\right|, \quad|\xi| \geqslant R_{2},
\end{aligned}
$$

а из $(25),(26)$ и (28) - для всех $p, 1 \leqslant p \leqslant m$, оценки

$$
\begin{aligned}
\left|A_{p}(\xi)\right| & \leqslant \sum_{q=1}^{2 m}\left|g_{q}(\xi)\right| \frac{\left|\Delta_{p+m, q}(\xi)\right|}{|\Delta(\xi)|} \\
& \leqslant 2 e^{|\xi|\left(\nu-\frac{\sigma}{2}-\frac{\sigma}{2 m}\right)} \sum_{q=1}^{2 m} C_{m+p, q}\left|g_{q}(\xi)\right| \\
& \leqslant 2 e^{|\xi|\left(\nu-\frac{\sigma}{2}\right)} \sum_{q=1}^{2 m} C_{p+m, q}\left|g_{q}(\xi)\right|, \quad|\xi| \geqslant R_{2} .
\end{aligned}
$$

Если в (29) положить $\nu=1-\varepsilon / 2, \sigma=\varepsilon$ при произвольно взятом $\varepsilon \in(0,1 / 2)$, то мы получим, что функции $B_{p}(\xi) e^{|\xi|(1-\varepsilon)} \in L_{2}\left(|\xi|>R_{2}\right)$ для всех $p, 1 \leqslant p \leqslant m$; полагая в (30) $\nu=3 \varepsilon / 2, \sigma=\varepsilon$ при $\varepsilon \in(0,1 / 2)$, получим, что функции $A_{p}(\xi) e^{-\varepsilon|\xi|} \in$ $L_{2}\left(|\xi|>R_{2}\right)$ при всех $p, 1 \leqslant p \leqslant m$ (число $R_{2}$, конечно, от $\varepsilon$ не зависит). Лемма 2 доказана.

Обозначим через $\chi_{R_{2}}(\xi)$ характеристическую функцию шара $\left\{|\xi|<R_{2}\right\}$. Равенство

$$
\widetilde{u}(\xi, y)=\chi_{R_{2}}(\xi) \widetilde{u}(\xi, y)+\left(1-\chi_{R_{2}}(\xi)\right) \widetilde{u}(\xi, y), \quad \xi \in \mathbb{R}_{n}, \quad 0<y<1,
$$

в силу (17) можно переписать $\left(R_{2} \geqslant R_{0}+1\right)$ в виде

$\widetilde{u}(\xi, y)=\chi_{R_{2}}(\xi) \widetilde{u}(\xi, y)+\sum_{i=1}^{m}\left(1-\chi_{R_{2}}(\xi)\right) A_{i}(\xi) w_{i}(\xi, y)+\left(1-\chi_{R_{2}}(\xi)\right) \sum_{i=1}^{m} B_{i}(\xi) v_{i}(\xi, y)$.

Следовательно,

$$
\begin{aligned}
u(x, y)= & \left(F^{-1} \widetilde{u}(\cdot, y)\right)(x)=\left(F^{-1} \chi_{R_{2}}(\cdot) \widetilde{u}(\cdot, y)\right)(x) \\
& +\sum_{i=1}^{m}\left(F^{-1}\left[\left(1-\chi_{R_{2}}(\cdot)\right) A_{i}(\cdot) w_{i}(\cdot, y)\right]\right)(x) \\
& +\left(F^{-1}\left(1-\chi_{R_{2}}(\cdot)\right) \sum_{i=1}^{m} B_{i}(\cdot) v_{i}(\cdot, y)\right)(x), \quad(x, y) \in \Pi .
\end{aligned}
$$


Функция

$$
\left(F^{-1} \chi_{R_{2}}(\cdot) \widetilde{u}(\cdot, y)\right)(x)=\frac{1}{(2 \pi)^{n / 2}} \int_{|\xi|<R_{2}} e^{i(x, \xi)} \widetilde{u}(\xi, y) d \xi
$$

очевидно, является решением уравнения (1) во всем пространстве $\mathbb{R}_{n+1}$ и, в частности, в П и принадлежит $W_{2}^{2 m}(\Pi)$. Функции

$$
\begin{aligned}
& \left(F^{-1}\left(1-\chi_{R_{2}}(\cdot)\right) A_{i}(\cdot) w_{i}(\cdot, y)\right)(x) \\
& \quad=\frac{1}{(2 \pi)^{n / 2}} \int_{|\xi|>R_{2}} e^{i(x, \xi)} A_{i}(\xi) w_{i}(\xi, y) d \xi, \quad i=1, \ldots, m
\end{aligned}
$$

являются решениями уравнения (1) в полупространстве $\left\{x \in \mathbb{R}_{n}, y>a\right\}$ и, в частности, в полосе $\left\{x \in \mathbb{R}_{n}, a<y<1\right\}$ и принадлежат $W_{2}^{2 m}\left(x \in \mathbb{R}_{n}, a<y<1\right)$ при любом $a \in(0,1)$, поскольку для любого такого $a$ производная произвольного порядка $p, p \geqslant 0$, по переменньм $(x, y)$ стояшей под знаком интеграла функции при всех $x \in \mathbb{R}_{n}$ и всех $y \geqslant a$ имеет в силу лемм 1 и 2 интегрируемую мажоранту $|g(\xi)| e^{-\frac{a}{2}|\xi|}|\xi|^{p}$, где $g(\xi)$ - некоторая, конечно, зависящая от $a$ функция из $L_{2}\left(|\xi|>R_{2}\right)$ (число $\varepsilon$ в (23) полагаем равным $a / 2$ ). Функция

$$
\begin{aligned}
& \left(F^{-1}\left(1-\chi_{R_{2}}(\cdot)\right) \sum_{i=1}^{m} B_{i}(\cdot) v_{i}(\cdot, y)\right)(x) \\
& \quad=\frac{1}{(2 \pi)^{n / 2}} \int_{|\xi|>R_{2}} e^{i(x, \xi)} \sum_{i=1}^{m} B_{i}(\xi) v_{i}(\xi, y) d \xi
\end{aligned}
$$

является решением уравнения (1) в полупространстве $\left\{x \in \mathbb{R}_{n}, y<1-a\right\}$ и, в частности, в полосе $\left\{x \in \mathbb{R}_{n}, 0<y<1-a\right\}$ и принадлежит $W_{2}^{2 m}\left(x \in \mathbb{R}_{n}\right.$, $0<y<1-a)$ при любом $a \in(0,1)$, поскольку для любого такого $a$ производная произвольного порядка $p, p \geqslant 0$, по переменньм $(x, y)$ стоящей под знаком интеграла функции при всех $x \in \mathbb{R}_{n}$ и всех $y \in[0,1-a]$ имеет в силу лемм $1^{\prime}$ и 2 интегрируемую мажоранту $e^{-\frac{a}{2}|\xi|}|\xi|^{p}|g(\xi)|$, где $g(\xi)$ - некоторая функция из $L_{2}\left(|\xi|>R_{2}\right)$ (число $\varepsilon$ в (24) полагаем равным $a / 2$ ). Так как функция $u(x, y)$, стоящая в левой части равенства (31), по условию и функции (32) и (33) по только что доказанному являются решениями уравнения (1) в П и принадлежат $W_{2}^{2 m}\left(x \in \mathbb{R}_{n}, a<y<1\right)$ при любом $a \in(0,1)$, то в силу (31) и функция (34) также является решением в П уравнения (1) и принадлежит $W_{2}^{2 m}\left(x \in \mathbb{R}_{n}, 0<y<1\right)$.

Равенство (31) можно переписать в виде

$$
u(x, y)=U(x, y)+\sum_{i=1}^{m} u_{i}(x, y), \quad(x, y) \in \Pi
$$

где $U(x, y)$ - сумма функций $(32)$ и $(34)$, а $u_{i}(x, y), i=1, \ldots, m,-$ функции из $(33)$. Таким образом, доказано следующее утверждение. 
Лемма 3. Принадлежсашее $W_{2}^{2 m}\left(x \in \mathbb{R}_{n}, a<y<1\right)$ при любом $a \in(0,1)$ решение $и(x, y)$ уравнения (1) мохсно представить в виде суммы (35) более простых решений $u_{1}(x, y), \ldots, u_{m}(x, y)$ и $U(x, y)$ этого уравнения, где $u_{i}(x, y) \in W_{2}^{2 m}\left(x \in \mathbb{R}_{n}, a<y<1\right), i=1, \ldots, m$, при любом $a \in(0,1)$ u

$$
\widetilde{u}_{i}(\xi, y)=A_{i}(\xi)\left(1-\chi_{R_{2}}(\xi)\right) w_{i}(\xi, y), \quad i=1, \ldots, m
$$

при некотором $R_{2}>0$, а $U(x, y) \in W_{2}^{2 m}\left(x \in \mathbb{R}_{n}, 0<y<1\right)$.

Установленное леммой 3 представление (35) исследуемого решения в виде суммы нескольких более простых решений будет играть в дальнейшем центральную роль. Приступим сначала к изучению отдельных слагаемых суммы (35).

Лемма 4. Пусть функиия $G(x, y) \in C\left([0,1] ; L_{2}\left(\mathbb{R}_{n}\right)\right)$. Тогда

$$
\begin{aligned}
& \sup _{0<y<1}\left\|P_{N} G(\cdot, y)\right\|_{L_{2}\left(\mathbb{R}_{n}\right)} \rightarrow 0 \quad \text { npu } \quad N \rightarrow \infty \\
& \sup _{0<y<1}\left\|T_{h} G(\cdot, y)\right\|_{W_{2}^{-1}\left(\mathbb{R}_{n}\right)} \rightarrow 0 \quad \text { npu } \quad|h| \rightarrow 0 .
\end{aligned}
$$

Из этой леммы, в частности, вытекает, что функция $U(x, y)$ из $(35)$, принадлежащая $C\left([0,1], L_{2}\left(\mathbb{R}_{n}\right)\right)$, удовлетворяет условиям $(37)$ и $(38)$.

ДокАЗАТЕЛЬСТво. Прежде всего проверим соотношения (37) и (38) для функции $G(x, y)$, не зависящей от $y: G(x, y) \equiv g(x)$. В этом случае

$$
\left\|P_{N} g\right\|_{L_{2}\left(\mathbb{R}_{n}\right)}^{2}=\int_{N<|\xi|<2 N}|\widetilde{g}(\xi)|^{2} d \xi \rightarrow 0 \text { при } N \rightarrow \infty,
$$

поскольку $\widetilde{g}(\xi) \in L_{2}\left(\mathbb{R}_{n}\right)$. Аналогично обстоит дело и со вторым соотношением: в силу неравенства $(9)$

$$
\left\|T_{h} g\right\|_{W_{2}^{-1}\left(\mathbb{R}_{n}\right)}^{2} \leqslant \int_{\mathbb{R}_{n}}|\widetilde{g}(\xi)|^{2} \sin ^{2} \frac{(h, \xi)}{2} d \xi \rightarrow 0 \text { при }|h| \rightarrow 0,
$$

поскольку $\widetilde{g}(\xi) \in L_{2}\left(\mathbb{R}_{n}\right)$.

Докажем теперь соотношение (37) в общем случае. Если оно места не имеет, то существуют $\varepsilon_{0}>0$ и последовательности $\left\{N_{k}, k=1,2, \ldots\right\}$ и $\left\{y_{k}, k=1,2, \ldots\right\}$, $N_{k} \rightarrow \infty$ при $k \rightarrow \infty, y_{k} \in[0,1], k=1,2, \ldots$, такие, что

$$
\left\|P_{N_{k}} G\left(\cdot, y_{k}\right)\right\|_{L_{2}\left(\mathbb{R}_{n}\right)} \geqslant \varepsilon_{0} \quad \text { для всех } k=1,2, \ldots
$$

Последовательность $\left\{y_{k}, k=1,2, \ldots\right\}$ можно, не умаляя общности, считать сходящейся: $y_{k} \rightarrow y_{0}$ при $k \rightarrow \infty, y_{0} \in[0,1]$. Поскольку

$$
\begin{aligned}
\left\|P_{N_{k}} G\left(\cdot, y_{k}\right)\right\|_{L_{2}\left(\mathbb{R}_{n}\right)} & \leqslant\left\|P_{N_{k}}\left(G\left(\cdot, y_{k}\right)-G\left(\cdot, y_{0}\right)\right)\right\|_{L_{2}\left(\mathbb{R}_{n}\right)}+\left\|P_{N_{k}} G\left(\cdot, y_{0}\right)\right\|_{L_{2}\left(\mathbb{R}_{n}\right)} \\
& \leqslant\left\|G\left(\cdot, y_{k}\right)-G\left(\cdot, y_{0}\right)\right\|_{L_{2}\left(\mathbb{R}_{n}\right)}+\left\|P_{N_{k}} G\left(\cdot, y_{0}\right)\right\|_{L_{2}\left(\mathbb{R}_{n}\right)}
\end{aligned}
$$

и поскольку при достаточно больших $k$ первое слагаемое правой части последнего неравенства в силу того, что $G(x, y) \in C\left([0,1] ; L_{2}\left(R_{n}\right)\right)$, может быть сделано 
сколь угодно малым, а второе слагаемое - сколь угодно мальм в силу только что доказанного соотношения (37) для функции $g(x)=G\left(x, y_{0}\right)$, то неравенство (39) несостоятельно.

Аналогично доказывается (38). Если (38) не имеет места, то сушествуют $\varepsilon_{0}>0$ и последовательности $\left\{h^{k} \in \mathbb{R}_{n}, k=1,2 \ldots\right\},\left\{y^{k}, k=1,2, \ldots\right\},\left|h^{k}\right| \rightarrow 0$, $y^{k} \rightarrow y_{0}, k \rightarrow \infty$, такие, что

$$
\left\|T_{h^{k}} G\left(\cdot, y^{k}\right)\right\|_{W_{2}^{-1}\left(\mathbb{R}_{n}\right)} \geqslant \varepsilon_{0}, \quad k=1,2, \ldots
$$

но этого быть не может, поскольку в силу (8)

$$
\begin{aligned}
\left\|T_{h^{k}} G\left(\cdot, y^{k}\right)\right\|_{W_{2}^{-1}\left(\mathbb{R}_{n}\right)} \leqslant & \left\|T_{h^{k}}\left(G\left(\cdot, y^{k}\right)-G\left(\cdot, y_{0}\right)\right)\right\|_{W_{2}^{-1}\left(\mathbb{R}_{n}\right)} \\
& +\left\|T_{h^{k}} G\left(\cdot, y_{0}\right)\right\|_{W_{2}^{-1}\left(\mathbb{R}_{n}\right)} \leqslant\left\|G\left(\cdot, y^{k}\right)-G\left(\cdot, y_{0}\right)\right\|_{L_{2}\left(\mathbb{R}_{n}\right)} \\
& +\left\|T_{h^{k}} G\left(\cdot, y_{0}\right)\right\|_{W_{2}^{-1}\left(\mathbb{R}_{n}\right)} \rightarrow 0 \quad \text { при } k \rightarrow \infty .
\end{aligned}
$$

Лемма доказана.

Рассмотрим слагаемое $u_{1}(x, y)$ в сумме $(35)$.

ЛЕмМа 5. Если и ${ }_{1}(x, y)$ из (35) $L_{2}$-ограниченная функиия, то

1) существует ее $L_{2}$-предел при $y \rightarrow+0$,

2) $\sup _{0<y<1}\left\|P_{N} u_{1}(\cdot, y)\right\|_{L_{2}\left(\mathbb{R}_{n}\right)} \rightarrow 0, N \rightarrow \infty$,

3) $\sup _{0<y<1}\left\|T_{h} u_{1}(\cdot, y)\right\|_{W_{2}^{-1}\left(\mathbb{R}_{n}\right)} \rightarrow 0, h \rightarrow 0$.

В силу $(36)$ и $(19)$ для всех $y \in[0,1]$ имеет место $\left(R_{2} \geqslant R_{1}\right)$ неравенство

$$
\begin{aligned}
\left\|u_{1}(\cdot, y)\right\|_{L_{2}\left(\mathbb{R}_{n}\right)}^{2} & =\int_{|\xi|>R_{2}}\left|A_{1}(\xi)\right|^{2}\left|w_{1}(\xi, y)\right|^{2} d \xi \\
& \geqslant \frac{1}{2} \int_{|\xi|>R_{2}}\left|A_{1}(\xi)\right|^{2} e^{-2 y|\xi|} d \xi \geqslant \frac{1}{2} \int_{N>|\xi|>R_{2}}\left|A_{1}(\xi)\right|^{2} e^{-2 y|\xi|} d \xi
\end{aligned}
$$

при любом $N>R_{2}$, т.е. для любого $y \in[0,1]$ и любого $N>R_{2}$

$$
\frac{1}{2} \int_{R_{2}<|\xi|<N}\left|A_{1}(\xi)\right|^{2} e^{-2 y|\xi|} d \xi \leqslant M^{2}
$$

где $M=\sup _{0<y<1}\left\|u_{1}(\cdot, y)\right\|_{L_{2}\left(\mathbb{R}_{n}\right)}<\infty$. Переходя в последнем неравенстве к пределу при $y \rightarrow+0$, получим, что для любого $N>R_{2}$

$$
\int_{R_{2}<|\xi|<N}\left|A_{1}(\xi)\right|^{2} d \xi \leqslant 2 M^{2}
$$

Это означает, что $A_{1}(\xi) \in L_{2}\left(|\xi|>R_{2}\right)$. Поскольку функция $w_{1}(\xi, y)$ непрерывна по $y \in[0,1]$ при всех $\xi,|\xi|>R_{2}$, и ограничена (cp. (19)), то на основании теоремы Лебега

$$
\left\|u_{1}\left(\cdot, y^{\prime}\right)-u_{1}\left(\cdot, y^{\prime \prime}\right)\right\|_{L_{2}\left(\mathbb{R}_{n}\right)}^{2}=\int_{|\xi|>R_{2}}\left|A_{1}(\xi)\right|^{2}\left|w_{1}\left(\xi, y^{\prime}\right)-w_{1}\left(\xi, y^{\prime \prime}\right)\right|^{2} d \xi \rightarrow 0
$$


при $y^{\prime}-y^{\prime \prime} \rightarrow 0$, т.е. $u_{1}(x, y) \in C\left([0,1] ; L_{2}\left(\mathbb{R}_{n}\right)\right)$. Утверждение 1$)$ леммы 5 доказано. Утверждения 2) и 3) этой леммы теперь вытекают из предыдушей леммы 4. Лемма 5 доказана.

Прежде чем приступить к изучению оставшихся слагаемых суммы в (35), докажем одно элементарное утверждение, которым в дальнейшем будем неоднократно пользоваться.

Лемма 6. Пусть $f(x) \in L_{1 \mathrm{loc}}\left(\mathbb{R}_{n}\right)$ u $f(x) \geqslant 0$ п.в. в $\mathbb{R}_{n}$. Eсли

$$
\sup _{N>0} \int_{N<|x|<2 N} f(x) d x<\infty
$$

mo

$$
\sup _{N>0} \frac{1}{N} \int_{|x|<N}|x| f(x) d x<\infty
$$

и при любом $\theta>0$

$$
\frac{f(x)}{\left(1+|x|^{2}\right)^{\theta}} \in L_{1}\left(\mathbb{R}_{n}\right)
$$

Ecлu

$$
\lim _{N \rightarrow \infty} \int_{N<|x|<2 N} f(x) d x=0
$$

mo u

$$
\lim _{N \rightarrow \infty} \frac{1}{N} \int_{|x|<N}|x| f(x) d x=0 .
$$

Ясно, что утверждения леммы будут доказаны, если для функции одной переменной

$$
\alpha(r)=\int_{|x|=r} f(x) d S_{x}, \quad r>0,
$$

принадлежашей при любом $R>0$ пространству $L_{1}(0, R)$, показать, что из неравенства

$$
\sup _{N>0} \int_{N}^{2 N} \alpha(r) d r<\infty
$$

вытекает неравенство

$$
\sup _{N>0} \frac{1}{N} \int_{0}^{N} r \alpha(r) d r<\infty
$$

и принадлежность при любом $\theta>0$ функции $\alpha(r) /\left(1+r^{2}\right)^{\theta}$ пространству $L_{1}(0, \infty)$, а из условия

$$
\lim _{N \rightarrow \infty} \int_{N}^{2 N} \alpha(r) d r=0
$$

вытекает, что

$$
\lim _{N \rightarrow \infty} \frac{1}{N} \int_{0}^{N} r \alpha(r) d r=0 .
$$

Рассмотрим при $N \geqslant 0$ неотрицательную функцию

$$
F(N)=\int_{0}^{N} r \alpha(r) d r, \quad N \geqslant 0
$$


и определим для любого целого $m \geqslant 1$ функцию

$$
G(m)=\int_{2^{m-1}}^{2^{m}} r \alpha(r) d r, \quad m \geqslant 1 .
$$

Из (40) вытекает, что

$$
G(m) \leqslant 2^{m} \int_{2^{m-1}}^{2^{m}} \alpha(r) d r \leqslant M \cdot 2^{m} \text { для любого } m \geqslant 1,
$$

где $M=\sup _{N>0} \int_{N}^{2 N} \alpha(r) d r$. Если же имеет место $\left(40^{\prime}\right)$, то по любому $\varepsilon>0$ найдется такое $m_{0}>0$, что

$$
G(m) \leqslant 2^{m} \varepsilon \text { для всех } m \geqslant m_{0} .
$$

Для произвольного $N>1$ найдем целое $m \geqslant 1$ такое, что $2^{m-1}<N \leqslant 2^{m}$ $(m=[\ln N / \ln 2]+1) ;$ из $(40)$ имеем неравенство

$$
\begin{aligned}
F(N) & =\left(\int_{0}^{1}+\int_{1}^{2}+\cdots+\int_{2^{m-1}}^{N}\right)(r \alpha(r)) d r \leqslant\left(\int_{0}^{1}+\cdots+\int_{2^{m-1}}^{2 m}\right)(r \alpha(r)) d r \\
& \leqslant \int_{0}^{1} r \alpha(r) d r+2 M+2^{2} M+\cdots+2^{m} M=C_{0}+\left(2+\cdots+2^{m}\right) M
\end{aligned}
$$

и, следовательно,

$$
\frac{F(N)}{N} \leqslant \frac{C_{0}+M\left(2+\cdots+2^{m}\right)}{2^{m-1}} \leqslant \frac{C_{0}}{2^{m-1}}+4 M,
$$

и тем самым имеет место (41). Если же выполняется $\left(40^{\prime}\right)$, то для $N>2^{m_{0}+1}$ $\left(m=[\ln N / \ln 2]+1>m_{0}+1\right)$ имеет место неравенство

$$
F(N) \leqslant\left(\int_{0}^{2^{m_{0}+1}}+\cdots+\int_{2^{m-1}}^{2^{m}}\right)(r \alpha(r)) d r \leqslant C_{1}+\left(2^{m_{0}+2}+\cdots+2^{m}\right) \varepsilon,
$$

откуда

$$
\frac{F(N)}{N} \leqslant \frac{C_{1}}{N}+\left(2+1+\cdots+2^{m_{0}-m+3}\right) \varepsilon \leqslant \frac{C_{1}}{N}+4 \varepsilon,
$$

и тем самым $F(N) / N \leqslant 5 \varepsilon$ для достаточно больших $N$, т.е. выполняется $(41)$.

Покажем, что из (40) вытекает принадлежность при любом $\theta>0$ функции $\alpha(r) /\left(1+r^{2}\right)^{\theta}$ пространству $L_{1}(0, \infty)$. Действительно, для любого $N>0$

$$
\begin{aligned}
& \int_{0}^{N} \frac{\alpha(r)}{\left(1+r^{2}\right)^{\theta}} d r=\int_{0}^{N} \frac{1}{r\left(1+r^{2}\right)^{\theta}} d\left(\int_{0}^{r} \rho \alpha(\rho) d \rho\right) \\
& \quad=\frac{1}{\left(1+N^{2}\right)^{\theta}} \frac{1}{N} \int_{0}^{N} \rho \alpha(\rho) d \rho+\int_{0}^{N}\left(\frac{1}{r} \int_{0}^{r} \rho \alpha(\rho) d \rho\right)\left(\frac{1+(2 \theta+1) r^{2}}{r\left(1+r^{2}\right)^{\theta+1}}\right) d r \\
& \quad \leqslant \sup _{N>0}\left(\frac{1}{N} \int_{0}^{N} \rho \alpha(\rho) d \rho\right)\left[\frac{1}{\left(1+N^{2}\right)^{\theta}}+(2 \theta+1) \int_{0}^{N} \frac{d r}{r\left(1+r^{2}\right)^{\theta}}\right] \leqslant \mathrm{const}
\end{aligned}
$$

в силу (41). Лемма доказана.

Рассмотрим теперь слагаемые $u_{s}(x, y), s=2, \ldots, m$, суммы в $(35)$. 
Лемма 7. Пусть иелое $s \in[2, m]$.

1) Для $L_{2}$-ограниченности функции $u_{s}(x, y)$ из (35) необходимо и достаточно выполнения условия

$$
\sup _{N>0} \int_{N<|\xi|<2 N} \frac{\left|A_{s}(\xi)\right|^{2}}{\left(1+|\xi|^{2}\right)^{s-1}} d \xi<\infty
$$

2) Для существования $L_{2}$-предела при $y \rightarrow 0$ функиии $u_{s}(x, y)$ (әтот предел непременно нулевой) необходимо и достаточно выполнения условия

$$
\lim _{N \rightarrow \infty} \int_{N<|\xi|<2 N} \frac{\left|A_{s}(\xi)\right|^{2}}{\left(1+|\xi|^{2}\right)^{s-1}} d \xi=0
$$

Прежде всего проверим, что если сушествует $L_{2}$-предел $f(x)$ функции $u_{s}(x, y)$ при $y \rightarrow 0$, то $f(x)=0$. Действительно, для любой функции $g(x)$, преобразование Фурье $\widetilde{g}(\xi)$ которой является финитной функцией, имеет место (см. (36) и лемму 1 ) соотношение

$$
\left(u_{s}(\cdot, y), g(\cdot)\right)_{L_{2}\left(\mathbb{R}_{n}\right)}=\left(A_{s}(\cdot) w_{s}(\cdot, y), \widetilde{g}(\cdot)\right)_{L_{2}\left(|\xi|>R_{2}\right)} \rightarrow 0 \text { при } y \rightarrow 0 .
$$

Это означает, что $(f(\cdot), g(\cdot))_{L_{2}\left(\mathbb{R}_{n}\right)}=0$, откуда в силу плотности в $L_{2}\left(\mathbb{R}_{n}\right)$ рассматриваемого множества функций $g(x)$ имеем $f=0$.

ЗАмечАниЕ. В точности так же доказывается и следующее утверждение, которое нам понадобится при доказательстве леммы 9: только нулевая функция может быть $L_{2}$-пределом функции $u_{2}(x, y)+\cdots+u_{m}(x, y)$.

ДоКАЗАТЕЛЬСтво ЛЕммы 7. Необходимость. Пусть при доказательстве необходимости в первом утверждении леммы

$$
\left\|u_{s}(\cdot, y)\right\|_{L_{2}\left(\mathbb{R}_{n}\right)}^{2}=\left\|A_{s}(\cdot) w_{s}(\cdot, y)\right\|_{L_{2}\left(|\xi|>R_{2}\right)}^{2} \leqslant M \quad \text { для всех } y \in(0,1),
$$

где $M$ - некоторая постоянная, и при любом $\varepsilon>0$ имеется $\delta=\delta(\varepsilon)>0$ такое, что

$$
\left\|u_{s}(\cdot, y)\right\|_{L_{2}\left(\mathbb{R}_{n}\right)}^{2}=\left\|A_{s}(\cdot) w_{s}(\cdot, y)\right\|_{L_{2}\left(|\xi|>R_{2}\right)}^{2} \leqslant \varepsilon \quad \text { для всех } y \in(0, \delta)
$$

в случае доказательства необходимости во втором утверждении. Докажем, что выполняются соотношения ( 42$)$ и $\left(42^{\prime}\right)$ соответственно.

В силу неравенства (19) леммы 1 для любого $N \geqslant R_{2}$ из (43) вытекает справедливое при всех $y \in(0,1)$ неравенство

$$
\begin{aligned}
M & \geqslant \int_{|\xi|>R_{2}}\left|A_{s}(\xi)\right|^{2}\left|w_{s}(\xi, y)\right|^{2} d \xi \geqslant \int_{N<|\xi|<2 N}\left|A_{s}(\xi)\right|^{2}\left|w_{s}(\xi, y)\right|^{2} d \xi \\
& \geqslant \frac{1}{(2(s-1) !)^{2}} \int_{N<|\xi|<2 N}\left|A_{s}(\xi)\right|^{2} y^{2(s-1)} e^{-2 y|\xi|} d \xi
\end{aligned}
$$


из этого неравенства при $y=1 / N$ вытекает неравенство

$$
\begin{aligned}
M & \geqslant \frac{1}{(2(s-1) !)^{2}} \int_{N<|\xi|<2 N}\left|A_{s}(\xi)\right|^{2} \frac{1}{N^{2(s-1)}} e^{-2 \frac{|\xi|}{N}} d \xi \\
& \geqslant \frac{1}{\left(2(s-1) ! e^{2}\right)^{2}} \int_{N<|\xi|<2 N} \frac{\left|A_{s}(\xi)\right|^{2}}{\left(1+|\xi|^{2}\right)^{s-1}} d \xi,
\end{aligned}
$$

из которого следует (42).

Если имеет место $\left(43^{\prime}\right)$, то приведенная вьше выкладка (точка $y=1 / N$ при всех достаточно больших $N, N>1 / \delta$, содержится в $(0, \delta))$ дает

$$
\varepsilon \geqslant \frac{1}{\left(2(s-1) ! e^{2}\right)^{2}} \int_{N<|\xi|<2 N} \frac{\left|A_{s}(\xi)\right|^{2}}{\left(1+|\xi|^{2}\right)^{s-1}} d \xi \text { при всех } N>\frac{1}{\delta},
$$

т.е. имеет место $\left(42^{\prime}\right)$.

Достаточность. Пусть выполнено условие (42) или условие $\left(42^{\prime}\right)$. Покажем, что функция

$$
\left\|u_{s}(\cdot, y)\right\|_{L_{2}\left(\mathbb{R}_{n}\right)}^{2}=\left\|A_{s}(\cdot) w_{s}(\cdot, y)\right\|_{L_{2}\left(|\xi|>R_{2}\right)}^{2}, \quad y \in(0,1)
$$

ограничена или соответственно стремится к нулю при $y \rightarrow 0$. Так как функция $w_{s}(\xi, y), s=1, \ldots, m$, зависит лишш от $|\xi|$ и $y$ (см. (13) и (16)), т.е. $w_{s}(\xi, y)=$ $w_{s}(|\xi|, y), s=1, \ldots, m$, то, как и в доказательстве леммы 6 , удобно ввести в рассмотрение функцию $\alpha_{s}(r), r \geqslant 0, s=1, \ldots, m$, равную нулю при $r<R_{2}$ и равную

$$
\int_{|\xi|=r}\left|A_{s}(\xi)\right|^{2} d S \text { при } r>R_{2}
$$

Очевидно, $\alpha_{s}(r) \in L_{1}(0, R)$ при любом $R>0$. В терминах функции $\alpha_{s}(r)$, $2 \leqslant s \leqslant m$, из условия

$$
\sup _{N>0} \int_{N}^{2 N} \frac{\alpha_{s}(r)}{\left(1+r^{2}\right)^{s-1}} d r<\infty
$$

нам нужно получить, что

$$
\sup _{0<y<1} \int_{0}^{\infty} \alpha_{s}(r)\left|w_{s}(r, y)\right|^{2} d r<\infty
$$

а из условия

$$
\lim _{N \rightarrow \infty} \int_{N}^{2 N} \frac{\alpha_{s}(r)}{\left(1+r^{2}\right)^{s-1}} d r=0
$$

- получить, что

$$
\lim _{y \rightarrow 0} \int_{0}^{\infty} \alpha_{s}(r)\left|w_{s}(r, y)\right|^{2} d r=0
$$

В силу леммы 1

$$
\begin{aligned}
\int_{0}^{\infty} \alpha_{s}(r)\left|w_{s}(r, y)\right|^{2} d r & \leqslant\left(\frac{2}{(s-1) !}\right)^{2} y^{2(s-1)} \int_{R_{2}}^{\infty} \alpha_{s}(r) e^{-2 r y} d r \\
& \leqslant\left(\frac{2}{(s-1) !}\right)^{2} y^{2(s-1)} \int_{1}^{\infty} \alpha_{s}(r) e^{-2 r y} d r
\end{aligned}
$$


(считаем, что $R_{2} \geqslant 1$ ). Интеграл, стояший в правой части (46), преобразуем следуюшим образом:

$$
\begin{aligned}
y^{2(s-1)} & \int_{1}^{\infty} \alpha_{s}(r) e^{-2 r y} d r=y^{2(s-1)} \int_{1}^{\infty} e^{-2 r y} \frac{r \alpha_{s}(r)}{\left(1+r^{2}\right)^{s-1}} \frac{\left(1+r^{2}\right)^{s-1}}{r} d r \\
& =y^{2(s-1)} \int_{1}^{\infty} e^{-2 r y} \frac{\left(1+r^{2}\right)^{s-1}}{r} d\left(\int_{1}^{r} \frac{\rho \alpha_{s}(\rho) d \rho}{\left(1+\rho^{2}\right)^{s-1}}\right) \\
& =-y^{2(s-1)} \int_{1}^{\infty}\left(\frac{1}{r} e^{-2 r y}\left(1+r^{2}\right)^{s-1}\right)^{\prime}\left(\int_{1}^{r} \frac{\rho \alpha_{s}(\rho) d \rho}{\left(1+\rho^{2}\right)^{s-1}}\right) d r \\
& =\int_{1}^{\infty}\left(\frac{1}{r} \int_{1}^{r} \frac{\rho \alpha_{s}(\rho) d \rho}{\left(1+\rho^{2}\right)^{s-1}}\right) \psi(r, y) d r,
\end{aligned}
$$

где

$$
\psi(r, y)=-y^{2(s-1)} r\left(e^{-2 r y} \frac{\left(1+r^{2}\right)^{s-1}}{r}\right)^{\prime} .
$$

Граничные члены в проведенном выше интегрировании по частям отсутствуют, поскольку, очевидно,

$$
\lim _{r \rightarrow 1} \frac{1}{r} e^{-2 r y}\left(1+r^{2}\right)^{s-1} \int_{1}^{r} \frac{\rho \alpha_{s}(\rho) d \rho}{\left(1+\rho^{2}\right)^{s-1}}=0
$$

и в силу леммы 6

$$
\lim _{r \rightarrow \infty} \frac{1}{r} e^{-2 r y}\left(1+r^{2}\right)^{s-1} \int_{1}^{r} \frac{\rho \alpha_{s}(\rho) d \rho}{\left(1+\rho^{2}\right)^{s-1}}=0 .
$$

Так как

$$
\begin{aligned}
|\psi(r, y)|= & r y^{2(s-1)} \mid-\frac{2 y}{r}\left(1+r^{2}\right)^{s-1} e^{-2 r y}-e^{-2 r y} \frac{\left(1+r^{2}\right)^{s-1}}{r^{2}} \\
& +2 e^{-2 r y}(s-1)\left(1+r^{2}\right)^{s-2} \mid \\
\leqslant & e^{-2 r y}\left(2 y^{2 s-1}\left(1+r^{2}\right)^{s-1}+\frac{\left(1+r^{2}\right)^{s-1}}{r} y^{2(s-1)}\right. \\
& \left.+2(s-1) r y^{2(s-1)}\left(1+r^{2}\right)^{s-2}\right)
\end{aligned}
$$

TO

$$
\begin{aligned}
\int_{1}^{\infty}|\psi(r, y)| d r \leqslant & \int_{1}^{\infty}\left(2^{s} y^{2 s-1} r^{2(s-1)}+2^{s-1} r^{2 s-3} y^{2(s-1)}\right. \\
& \left.+2^{s-1}(s-1) y^{2(s-1)} r^{2 s-3}\right) e^{-2 r y} d r \\
\leqslant & \int_{0}^{\infty}\left(2^{s} \rho^{2 s-2}+s 2^{s-1} \rho^{2 s-3}\right) e^{-2 \rho} d \rho=C_{0}
\end{aligned}
$$

для всех $y \in(0,1)$ (напомним, что $s \geqslant 2$ ). Поэтому в продолжение (47) имеем

$$
\begin{aligned}
y^{2(s-1)} \int_{1}^{\infty} \alpha_{s}(r) e^{-2 r y} d y & \leqslant \int_{1}^{\infty}\left(\frac{1}{r} \int_{1}^{r} \frac{\rho \alpha_{s}(\rho) d \rho}{\left(1+\rho^{2}\right)^{s-1}}\right)|\psi(r, y)| d r \\
& \leqslant \sup _{r>1}\left(\frac{1}{r} \int_{1}^{\infty} \frac{\rho \alpha_{s}(\rho) d \rho}{\left(1+\rho^{2}\right)^{s-1}}\right) C_{0}
\end{aligned}
$$

для всех $y \in(0,1)$, откуда в силу леммы 6 следует $(45)$. 
Если же речь идет о втором утверждении леммы, то правую часть в (46) оценим следующим образом: из $\left(44^{\prime}\right)$ в силу леммы 6 по любому $\varepsilon>0$ найдем $N^{\prime}>1$ такое, что

$$
\frac{1}{N} \int_{0}^{N} \frac{r \alpha_{s}(r) d r}{\left(1+r^{2}\right)^{s-1}}<\varepsilon \text { для всех } N \geqslant N^{\prime}
$$

Тогда (см. (47))

$$
\begin{aligned}
& \left|\int_{1}^{\infty}\left(\frac{1}{r} \int_{1}^{r} \frac{\rho \alpha_{s}(\rho) d \rho}{\left(1+\rho^{2}\right)^{s-1}}\right) \psi(r, y) d r\right| \leqslant\left|\int_{1}^{N^{\prime}}\left(\frac{1}{r} \int_{1}^{r} \frac{\rho \alpha_{s}(\rho) d \rho}{\left(1+\rho^{2}\right)^{s-1}}\right) \psi(r, y) d r\right| \\
& \quad+\left|\int_{N^{\prime}}^{\infty}\left(\frac{1}{r} \int_{1}^{r} \frac{\rho \alpha_{s}(\rho) d \rho}{\left(1+\rho^{2}\right)^{s-1}}\right) \psi(r, y) d r\right| \\
& \leqslant\left|\int_{1}^{N^{\prime}}\right| \frac{1}{r} \int_{1}^{r} \frac{\rho \alpha_{s}(\rho) d \rho}{\left(1+\rho^{2}\right)^{s-1}}|\psi(r, y)| d r\left|+\varepsilon \int_{N^{\prime}}^{\infty}\right| \psi(r, y) \mid d r \\
& \leqslant \int_{1}^{N^{\prime}}\left|\frac{1}{r} \int_{1}^{r} \frac{\rho \alpha_{s}(\rho) d \rho}{\left(1+\rho^{2}\right)^{s-1}}\right||\psi(r, y)| d r+C_{0} \varepsilon \leqslant \varepsilon\left(C_{0}+1\right)
\end{aligned}
$$

поскольку первое слагаемое в силу (48) $\left(\lim _{y \rightarrow 0}|\psi(r, y)|=0\right)$ может быть сделано меньшим $\varepsilon$. Лемма доказана.

Лемма 8. Пусть $2 \leqslant s \leqslant m$. Следующие три утверждения әквивалентны.

1) $\left\|u_{s}(\cdot, y)\right\|_{L_{2}\left(\mathbb{R}_{n}\right)} \rightarrow 0, y \rightarrow 0$.

2) $\sup _{0<y<1}\left\|P_{N} u_{s}(\cdot, y)\right\|_{L_{2}\left(\mathbb{R}_{n}\right)} \rightarrow 0, N \rightarrow \infty$.

3) $\sup _{0<y<1}\left\|T_{h} u_{s}(\cdot, y)\right\|_{W_{2}^{-1}\left(\mathbb{R}_{n}\right)} \rightarrow 0, h \rightarrow 0$.

То, что из утверждения 1) вытекают утверждения 2) и 3), есть прямое следствие леммы 4; поэтому для доказательства леммы 8 нам следует проверить обратное: что из 2) вытекает 1) и что из 3) вытекает 1). Утверждения 1), 2) и 3 ) в терминах преобразования Фурье имеют соответственно вид (см. (36)):

$$
\begin{gathered}
\left\|A_{s}(\cdot) w_{s}(\cdot, y)\right\|_{L_{2}\left(|\xi|>R_{2}\right)} \rightarrow 0, \quad y \rightarrow 0, \\
\sup _{0<y<1}\left\|\zeta_{N, 2 N}(\cdot) A_{s}(\cdot) w_{s}(\cdot, y)\right\|_{L_{2}\left(|\xi|>R_{2}\right)} \rightarrow 0, \quad N \rightarrow \infty, \\
\sup _{0<y<1}\left\|\frac{A_{s}(\cdot) w_{s}(\cdot, y) \sin ^{2} \frac{(h, \cdot)}{2}}{\left(1+|\cdot|^{2}\right)^{1 / 2}|h|}\right\|_{L_{2}\left(|\xi|>R_{2}\right)} \rightarrow 0, \quad h \rightarrow 0,
\end{gathered}
$$

где $\zeta_{N, 2 N}(\xi)$ - характеристическая функция шарового слоя $\{N<|\xi|<2 N\}$.

Заметим, что соотношение (49) в силу предыдушей леммы эквивалентно соотношению

$$
\lim _{N \rightarrow \infty} \int_{N<|\xi|<2 N} \frac{\left|A_{s}(\xi)\right|^{2}}{\left(1+|\xi|^{2}\right)^{s-1}} d \xi=0
$$

Докажем сначала, что из (50) вытекает $\left(49^{\prime}\right)$, что эквивалентно соответствию $2) \rightarrow 1)$. Из $(50)$ в силу леммы 1 имеем, что по любому $\varepsilon>0$ найдется $N_{0}$ (будем считать $N_{0}>\max \left(1 ; R_{2}\right)$, где $R_{2}$ - число из леммы 3$)$ такое, что

$$
\int_{N<|\xi|<2 N}\left|A_{s}(\xi)\right|^{2} y^{2(s-1)} e^{-2|\xi| y} d \xi \leqslant \varepsilon \quad \text { для всех } N \geqslant N_{0} \quad \text { и } y \in(0,1) .
$$


Подставляя в это неравенство $y=1 / N$, получим

$$
\begin{aligned}
\varepsilon & \geqslant \int_{N<|\xi|<2 N}\left|A_{s}(\xi)\right|^{2} \frac{1}{N^{2(s-1)}} e^{-\frac{2|\xi|}{N}} d \xi \\
& \geqslant e^{-4} \int_{N<|\xi|<2 N} \frac{\left|A_{s}(\xi)\right|^{2} 2^{2(s-1)}}{|\xi|^{2(s-1)}} d \xi \\
& \geqslant 2^{2(s-1)} e^{-4} \int_{N<|\xi|<2 N} \frac{\left|A_{s}(\xi)\right|^{2}}{\left(1+|\xi|^{2}\right)^{s-1}} d \xi
\end{aligned}
$$

откуда вытекает $\left(49^{\prime}\right)$.

Покажем теперь, что из (51) также вытекает 1$)$. Пусть при произвольно взятом $\varepsilon>0$ число $\delta=\delta(\varepsilon)>0$ таково, что

$$
\varepsilon \geqslant \int_{\mathbb{R}_{n}} \frac{\left|A_{s}(\xi)\right|^{2}\left|w_{s}(\xi, y)\right|^{2}}{\left(1+|\xi|^{2}\right)|h|^{2}} \sin ^{4} \frac{(\xi, h)}{2} d \xi \quad \text { для всех } h, \quad|h| \leqslant \delta .
$$

Рассмотрим сначала случай $n>1$. Полагая $h=|h| h^{0}, \xi=|\xi| \xi^{0}$, где $h^{0}=h /|h|$ при $|h|>0, \xi^{0}=\xi /|\xi|$ при $|\xi|>0$, проинтегрируем неравенство (52) по единичной сфере $\left\{\left|h^{0}\right|=1\right\}$ (считая $\left.|h| \leqslant \delta\right)$ :

$$
\varepsilon \omega_{n} \geqslant \int_{\mathbb{R}_{n}} \frac{\left|A_{s}(\xi)\right|^{2}\left|w_{s}(\xi, y)\right|^{2}}{\left(1+|\xi|^{2}\right)|h|^{2}} \int_{\left|h^{0}\right|=1} \sin ^{4} \frac{|\xi||h|\left(\xi^{0}, h^{0}\right)}{2} d S_{h^{0}} d \xi
$$

где $\omega_{n}$ - площадь единичной сферы в $\mathbb{R}_{n}$. Поскольку при

$$
\begin{aligned}
& h_{1}^{0}=\cos \varphi_{1}, \\
& h_{2}^{0}=\sin \varphi_{1} \cos \varphi_{2} \text {, } \\
& \text {.............................. } \\
& h_{n-1}^{0}=\sin \varphi_{1} \cdots \sin \varphi_{n-2} \cos \varphi_{n-1} \text {, } \\
& h_{n}^{0}=\sin \varphi_{1} \cdots \sin \varphi_{n-2} \sin \varphi_{n-1},
\end{aligned}
$$

$0<\varphi_{1}<\pi, \ldots, 0<\varphi_{n-2}<\pi, 0<\varphi_{n-1}<2 \pi$, где $\varphi_{1}$ - угол между векторами $h^{0}$ и $\xi^{0}$ (северный полюс единичной сферы $\left\{\left|h^{0}\right|=1\right\}$ помешен в точку $\left.\xi^{0}\right)$, то

$$
\begin{aligned}
\int_{\left|h^{0}\right|=1} & \sin ^{4} \frac{|h||\xi|\left(h^{0}, \xi^{0}\right)}{2} d S_{h^{0}}=\int_{0}^{2 \pi} d \varphi_{n-1} \int_{0}^{\pi} \sin \varphi_{n-2} d \varphi_{n-2} \\
& \times \cdots \times \int_{0}^{\pi} \sin ^{n-3} \varphi_{2} d \varphi_{2} \int_{0}^{\pi} \sin ^{n-2} \varphi_{1} \sin ^{4} \frac{|h||\xi| \cos \varphi_{1}}{2} d \varphi_{1} \\
= & \omega_{n}^{\prime} \int_{0}^{1}\left(1-u^{2}\right)^{\frac{n-3}{2}} \sin ^{4} \frac{|\xi||h| u}{2} d u
\end{aligned}
$$

где

$$
\omega_{n}^{\prime}=2 \int_{0}^{2 \pi} d \varphi_{n-1} \int_{0}^{\pi} \sin \varphi_{n-2} d \varphi_{n-2} \cdots \int_{0}^{\pi} \sin ^{n-3} \varphi_{2} d \varphi_{2} \neq 0
$$


при $n>2$, а при $n=2 \omega_{2}^{\prime}=4$. Следовательно, из (53) имеем

$$
\begin{aligned}
\varepsilon \frac{\omega_{n}}{\omega_{n}^{\prime}} & \geqslant \int_{\mathbb{R}_{n}} d \xi \int_{0}^{1} \frac{\left|A_{s}(\xi)\right|^{2}\left|w_{s}(\xi, y)\right|^{2}}{\left(1+|\xi|^{2}\right)|h|^{2}} \sin ^{4} \frac{|\xi||h| u}{2}\left(1-u^{2}\right)^{(n-3) / 2} d u \\
& \geqslant \int_{1<|\xi||h|<2} d \xi \int_{\pi / 6}^{\pi / 4} \frac{\left|A_{s}(\xi)\right|^{2}\left|w_{s}(\xi, y)\right|^{2}}{\left(1+|\xi|^{2}\right)|h|^{2}} \sin ^{4} \frac{|\xi||h| u}{2}\left(1-u^{2}\right)^{(n-3) / 2} d u .
\end{aligned}
$$

Поскольку $\frac{|\xi||h| u}{2} \in\left[\frac{\pi}{12}, \frac{\pi}{4}\right]$ при $|\xi||h| \in[1,2]$ и $u \in\left[\frac{\pi}{6}, \frac{\pi}{4}\right]$, то

$$
\begin{aligned}
\sin ^{4} \frac{|\xi||h| u}{2} & =\left(\frac{\sin \frac{|\xi||h| u}{2}}{\frac{|\xi||h| u}{2}}\right)^{2} \frac{|\xi|^{2}|h|^{2} u^{2}}{4} \sin ^{2} \frac{|\xi||h| u}{2} \\
& \geqslant\left(\frac{\sin \frac{\pi}{4}}{\frac{\pi}{4}}\right)^{2} \frac{|\xi|^{2}|h|^{2} u^{2}}{4}\left(\sin \frac{\pi}{12}\right)^{2} \geqslant 2\left(\frac{\sin \frac{\pi}{12}}{\pi}\right)^{2}|\xi|^{2}|h|^{2} u^{2} .
\end{aligned}
$$

Поэтому при $|h| \leqslant \delta$

$$
\begin{aligned}
\varepsilon \frac{\omega_{n}}{\omega_{n}^{\prime}} \geqslant & 2\left(\frac{\sin \frac{\pi}{12}}{\pi}\right)^{2} \int_{\frac{1}{|h|}<|\xi|<\frac{2}{|h|}} \frac{|\xi|^{2}\left|A_{s}(\xi)\right|^{2}\left|w_{s}(\xi, y)\right|^{2}}{1+|\xi|^{2}} d \xi \\
& \times \int_{\frac{\pi}{6}}^{\frac{\pi}{4}} u^{2}\left(1-u^{2}\right)^{\frac{n-3}{2}} d u \geqslant \omega_{n}^{\prime \prime} \int_{\frac{1}{|h|}<|\xi|<\frac{2}{|h|}}\left|A_{s}(\xi)\right|^{2}\left|w_{s}(\xi, y)\right|^{2} d \xi
\end{aligned}
$$

где

$$
\omega_{n}^{\prime \prime}=\int_{\frac{\pi}{6}}^{\frac{\pi}{4}} u^{2}\left(1-u^{2}\right)^{\frac{n-3}{2}} d u \cdot \frac{\sin ^{2} \frac{\pi}{12}}{\pi^{2}}>0
$$

(считаем, что $|h| \leqslant 1)$. Это означает, что для всех $y \in(0,1)$

$$
\int_{N<|\xi|<2 N}\left|A_{s}(\xi)\right|^{2}\left|w_{s}(\xi, y)\right|^{2} d \xi \leqslant \varepsilon \frac{\omega_{n}}{\omega_{n}^{\prime} \omega_{n}^{\prime \prime}}=\text { const } \cdot \varepsilon
$$

при $N=1 /|h| \geqslant 1 / \delta(\varepsilon)$, т.е. выполняется соотношение $(50)$, из которого мы только что выше вьвели (49).

В случае $n=1$ доказательство несколько короче. В неравенстве (52) в этом случае число $h$ можно считать в силу четности по $h$ подынтегральной функции положительным: $h>0$. Далее,

$$
\begin{aligned}
\varepsilon & \geqslant \int_{\mathbb{R}_{1}} \frac{\left|A_{s}(\xi)\right|^{2}\left|w_{s}(\xi, y)\right|^{2}}{\left(1+\xi^{2}\right) h^{2}} \sin ^{4} \frac{\xi h}{2} d \xi \\
& \geqslant(\sin 1)^{2}\left(\sin \frac{1}{2}\right)^{2} \int_{\frac{1}{2}<\frac{\xi h}{2}<1} \frac{\left|A_{s}(\xi)\right|^{2}\left|w_{s}(\xi, y)\right|^{2}}{\left(1+\xi^{2}\right) h^{2}} \frac{\xi^{2} h^{2}}{4} d \xi \\
& \geqslant \frac{\left(\sin 1 \cdot \sin \frac{1}{2}\right)^{2}}{8} \int_{\frac{1}{h}<\xi<\frac{2}{h}}\left|A_{s}(\xi)\right|^{2}\left|w_{s}(\xi, y)\right|^{2} d \xi,
\end{aligned}
$$

если $h \leqslant 1$. Поэтому, как и в обшем случае, имеет место (50) и тем самьм $(49)$. Лемма доказана.

Представление решения уравнения (1) в виде суммы (35) обладает следующим важньм свойством. Входящие в (35) слагаемые в определенном смысле независимы: они ведут себя около границы настолько различным образом, что не могут компенсировать друг друга. Эта независимость слагаемых в (35) выражается следующими леммами. 


\section{Лемма 9.}

1) Решение и( $x, y)$ уравнения (1) $L_{2}$-ограничено тогда и только тогда, когда $L_{2}$-ограничено каждое слагаемое суммы (35).

2) Решение $и(x, y)$ уравнения (1) имеет $L_{2}$-предел при $y \rightarrow 0$ тогда $u$ только тогда, когда $L_{2}$-предель при у $\rightarrow 0$ существуют у каждого слагаемого суммы (35).

Естественно, что каждое из этих утверждений нужно доказывать только в одну сторону: из ограниченности или сушествования предела решения $u(x, y)$ следует получить ограниченность или соответственно существование предела каждого слагаемого в (35).

Пусть $u(x, y) \quad L_{2}$-ограничено. Поскольку функция $U(x, y) L_{2}$-ограничена, то $L_{2}$-ограничена и сумма $u_{1}(x, y)+\cdots+u_{m}(x, y)$, что эквивалентно в силу $(36)$ неравенству

$$
\left\|\sum_{i=1}^{m} A_{i}(\cdot) w_{i}(\cdot, y)\right\|_{L_{2}\left(|\xi|>R_{2}\right)}^{2} \leqslant M, \quad y \in(0,1),
$$

при некоторой постоянной $M>0$. Из этого неравенства немедленно вытекает нужное неравенство для слагаемого $u_{1}(x, y)$ в сумме (35):

$$
\left\|u_{1}(\cdot, y)\right\|_{L_{2}\left(\mathbb{R}_{n}\right)}^{2}=\left\|A_{1}(\cdot) w_{1}(\cdot, y)\right\|_{L_{2}\left(|\xi|>R_{2}\right)}^{2} \leqslant M_{1}, \quad y \in(0,1),
$$

с некоторой постоянной $M_{1}>0$. Для получения $\left(55_{1}\right)$ в неравенстве

$$
\left\|\sum_{i=1}^{m} A_{i}(\cdot) w_{i}(\cdot, y)\right\|_{L_{2}\left(R_{2}<|\xi|<N\right)}^{2} \leqslant M, \quad y \in(0,1)
$$

которое для любого $N>R_{2}$ вытекает из (54), перейдем к пределу при $y \rightarrow 0$. В результате получим, что при любом $N>R_{2}$ справедливо неравенство $\left\|A_{1}(\cdot)\right\|_{L_{2}\left(R_{2}<|\xi|<N\right)}^{2} \leqslant M$, т.е. $A_{1}(\xi) \in L_{2}\left(|\xi|>R_{2}\right)$. Неравенство $\left(55_{1}\right)$ после этого следует из непрерывности и ограниченности (см. лемму 1 ) функции $w_{1}(\xi, y)$.

Из (54) и $\left(55_{1}\right)$ имеем

$$
\left\|\sum_{i=2}^{m} A_{i}(\cdot) w_{i}(\cdot, y)\right\|_{L_{2}\left(|\xi|>R_{2}\right)}^{2} \leqslant M^{\prime}, \quad y \in(0,1)
$$

где $M^{\prime}=2\left(M+M_{1}\right)$.

Покажем теперь, что из (56) вытекает ограниченность каждого из оставшихся слагаемых в (35):

$$
\left\|A_{i}(\cdot) w_{i}(\cdot, y)\right\|_{L_{2}\left(|\xi|>R_{2}\right)}^{2} \leqslant M_{i}, \quad y \in(0,1), \quad i=2, \ldots, m,
$$

где $M_{i}>0$ - некоторые постоянные, $i=2, \ldots, m$.

Для этого прежде всего отметим, что из (56) следует справедливость при любом $N>R_{2}$ неравенства

$$
\int_{N<|\xi|<2 N}\left|\sum_{i=2}^{m} A_{i}(\xi) w_{i}(\xi, y)\right|^{2} d \xi \leqslant M^{\prime}, \quad y \in(0,1) ;
$$


разделим это неравенство на $y^{2}$, проинтегрируем полученное неравенство по $y$ от $y \in(0,1)$ до 1

$$
\int_{y}^{1} d \eta \int_{N<|\xi|<2 N}\left|\sum_{i=2}^{m} A_{i}(\xi) \frac{w_{i}(\xi, \eta)}{\eta}\right|^{2} d \xi \leqslant M^{\prime} \int_{y}^{1} \frac{d \eta}{\eta^{2}} \leqslant \frac{M^{\prime}}{y}, \quad y \in(0,1)
$$

и положим в последнем неравенстве $y=1 / N$ (считаем, что $\left.R_{2} \geqslant 1\right)$. В результате получим

$$
\begin{aligned}
M^{\prime} N & \geqslant \int_{N<|\xi|<2 N} \int_{1 / N}^{1}\left|\sum_{i=2}^{m} A_{i}(\xi) \frac{w_{i}(\xi, \eta)}{\eta}\right|^{2} d \eta d \xi \\
& =\left.\left.\int_{N<|\xi|<2 N} \int_{1 / N}^{1}\left|\sum_{i=2}^{m} B_{i}(\xi) \frac{w_{i}(\xi, \eta)}{\eta}\right| \xi\right|^{i-1}\right|^{2} d \eta d \xi
\end{aligned}
$$

где

$$
B_{i}(\xi)=\frac{A_{i}(\xi)}{|\xi|^{i-1}}, \quad|\xi| \neq 0, \quad i=2, \ldots, m
$$

Покажем, что квадратичная форма

$$
\left.\left.\int_{1 / N}^{1}\left|\sum_{i=2}^{m} B_{i} \frac{w_{i}(\xi, \eta)}{\sqrt{N} \eta}\right| \xi\right|^{i-1}\right|^{2} d \eta
$$

в $(m-1)$-мерном комплексномпространстве векторов $B=\left(B_{2}, \ldots, B_{m}\right)$ при всех $\xi$, $N<|\xi|<2 N$, и всех $N \geqslant R_{2}$ положительно определена, т.е. число

$$
\gamma_{0}=\left.\left.\inf _{\mathfrak{N}} \int_{1 / N}^{1}\left|\sum_{i=2}^{m} B_{i} \frac{w_{i}(\xi, \eta)}{\eta \sqrt{N}}\right| \xi\right|^{i-1}\right|^{2} d \eta>0
$$

где $\mathfrak{N}=\left\{N \geqslant R_{2},\|B\|=\left(\left|B_{2}\right|^{2}+\cdots+\left|B_{m}\right|^{2}\right)^{1 / 2}=1, N \leqslant|\xi| \leqslant 2 N\right\}$-множество $(m+n)$-мерного пространства переменных $N, B_{2}, \ldots, B_{m}, \xi_{1}, \ldots, \xi_{n}$.

Очевидно, что $\gamma_{0} \geqslant 0$. Доказательство неравенства (59) проведем от противного: предположим, что $\gamma_{0}=0$. Тогда существуют такие последовательности $\left\{N_{k}, k=1,2, \ldots\right\},\left\{B^{k}=\left\{B_{2}^{k}, \ldots, B_{m}^{k}\right\}, k=1,2, \ldots\right\},\left\{\xi^{k}=\left(\xi_{1}^{k}, \ldots, \xi_{n}^{k}\right)\right.$, $k=1,2, \ldots\}$, что

$$
\begin{aligned}
& \left.\left.\int_{1 / N_{k}}^{1}\left|\sum_{i=2}^{m} B_{i}^{k} \frac{w_{i}\left(\xi^{k}, \eta\right)}{\sqrt{N_{k}} \eta}\right| \xi^{k}\right|^{i-1}\right|^{2} d \eta \\
& \quad=\left.\left.\int_{\left|\xi^{k}\right| / N_{k} \mid}^{\left|\xi^{k}\right|}\left|\sum_{i=2}^{m} B_{i}^{k} \frac{w_{i}\left(\xi^{k}, \zeta /\left|\xi^{k}\right|\right)}{\sqrt{N_{k}} \frac{\zeta}{\left|\xi^{k}\right|}}\right| \xi^{k}\right|^{i-1}\right|^{2} \frac{d \zeta}{\left|\xi^{k}\right|} \\
& \quad=\left.\left.\frac{\left|\xi^{k}\right|}{N_{k}} \int_{\left|\xi^{k}\right| / N_{k}}^{\left|\xi^{k}\right|}\left|\sum_{i=2}^{m} B_{i}^{k} \frac{w_{i}\left(\xi^{k}, \zeta /\left|\xi^{k}\right|\right)}{\zeta}\right| \xi^{k}\right|^{i-1}\right|^{2} d \zeta \rightarrow 0 \text { при } k \rightarrow \infty
\end{aligned}
$$

При этом, не умаляя общности, можно считать последовательности $\left\{B^{k}, k=\right.$ $1,2, \ldots\}$ и $\left\{\left|\xi^{k}\right| / N_{k}, k=1,2, \ldots\right\}$ сходящимися, т.е. считать, что сушествуют такой вектор $B^{0}=\left(B_{2}^{0}, \ldots, B_{m}^{0}\right),\left\|B^{0}\right\|=1$, и такое число $\alpha \in[1,2]$, что $B^{k} \rightarrow B^{0}$ и 
$\left|\xi^{k}\right| / N_{k} \rightarrow \alpha$ при $k \rightarrow \infty$, а последовательность $\left\{N_{k}, k=1,2, \ldots\right\}$ или а) сходится к некоторому числу $\beta: N_{k} \rightarrow \beta \geqslant R_{2}$ при $k \rightarrow \infty$, или б) $N_{k} \rightarrow \infty$ при $k \rightarrow \infty$; в случае а) можно дополнительно считать, что вектор $\xi^{k}=\left(\xi_{1}^{k}, \ldots, \xi_{n}^{k}\right) \rightarrow \xi^{0}=$ $\left\{\xi_{1}^{0}, \ldots, \xi_{n}^{0}\right\}$ при $k \rightarrow \infty$. В случае а) из (60) имеем равенство

$$
\alpha \int_{\alpha}^{\alpha \beta}\left|\sum_{i=2}^{m} B_{i}^{0} \frac{w_{i}\left(\xi^{0}, \zeta /(\alpha \beta)\right)}{\zeta}(\alpha \beta)^{i-1}\right|^{2} d \zeta=0,
$$

которое невозможно в силу линейной независимости системы $\left\{w_{i}\left(\xi^{0}, \zeta /(\alpha \beta)\right)\right.$, $i=2, \ldots, m\}$. В случае б) из равенства (60) с помощью леммы 1 заключаем, что

$$
\alpha \int_{\alpha}^{\infty}\left|\sum_{i=2}^{\infty} B_{i}^{0} \frac{e^{-\zeta} \zeta^{i-1}}{(i-1) !}\right|^{2} d \zeta=0
$$

чего также не может быть в силу линейной независимости системы функций $\left\{\zeta e^{-\zeta}, \zeta^{2} e^{-\zeta}, \ldots, \zeta^{m-1} e^{-\zeta}\right\}$ на множестве $\alpha \leqslant \zeta<\infty$.

Итак, неравенство (59) доказано, а вместе с ним доказано и неравенство

$$
\left.\left.\int_{1 / N}^{1}\left|\sum_{i=2}^{m} B_{i} \frac{w_{i}(\xi, \eta)}{\sqrt{N} \eta}\right| \xi\right|^{i-1}\right|^{2} d \eta \geqslant \gamma_{0} \sum_{i=2}^{m}\left|B_{i}\right|^{2}
$$

для всех $N \geqslant R_{2}$, всех $\xi, N \leqslant|\xi| \leqslant 2 N$, и любого вектора $B=\left(B_{2}, \ldots, B_{m}\right)$. Следовательно, из (58) получаем неравенство

$$
\begin{aligned}
M^{\prime} & \geqslant \gamma_{0} \int_{N<|\xi|<2 N} \sum_{i=2}^{m}\left|B_{i}(\xi)\right|^{2} d \xi=\gamma_{0} \int_{N<|\xi|<2 N} \sum_{i=2}^{m} \frac{\left.A_{i}(\xi)\right|^{2}}{|\xi|^{2(i-1)}} d \xi \\
& \geqslant \gamma_{0} \int_{N<|\xi|<2 N} \sum_{i=2}^{m} \frac{\left|A_{i}(\xi)\right|^{2}}{\left(1+|\xi|^{2}\right)^{i-1}} d \xi \geqslant \gamma_{0} \int_{N<|\xi|<2 N} \frac{\left|A_{i}(\xi)\right|^{2}}{\left(1+|\xi|^{2}\right)^{i-1}} d \xi
\end{aligned}
$$

справедливое для любого $i, i=2, \ldots, m$. Для доказательства первой части леммы теперь остается воспользоваться первым утверждением леммы 7 .

Пусть теперь функция $u(x, y)$ имеет $L_{2}$-предел при $y \rightarrow 0$. Тогда она $L_{2}$-ограничена и по только что доказанному $L_{2}$-ограничено, в частности, слагаемое $u_{1}(x, y)$ в равенстве (35). В силу первого утверждения леммы 5 функция $u_{1}(x, y)$ имеет $L_{2}$-предел при $y \rightarrow 0$. Поскольку функция $U(x, y)$ также имеет $L_{2}$-предел при $y \rightarrow 0$, то $L_{2}$-предел имеет и сумма $u_{2}(x, y)+\cdots+u_{m}(x, y)$, причем $L_{2}$-пределом этой суммы в силу замечания при доказательстве леммы 7 является нулевая функция. Это означает, что по любому $\varepsilon>0$ найдется такое $\delta=\delta(\varepsilon)>0$, что

$$
\left\|u_{2}(\cdot, y)+\cdots+u_{m}(\cdot, y)\right\|_{L_{2}\left(\mathbb{R}_{n}\right)}^{2}=\left\|\sum_{i=2}^{m} A_{i}(\cdot) w_{i}(\cdot, y)\right\|_{L_{2}\left(|\xi|>R_{2}\right)}^{2} \leqslant \varepsilon
$$

для всех $y \in(0, \delta)$. Это неравенство совпадает с неравенством (56), в котором $M^{\prime}=\varepsilon$, но которое должно выполняться не для всех $y \in(0,1)$, а лишь для $y \in(0, \delta)$. Повторяя рассуждения, приведшие выше к неравенствам (61), получим, что для всех $N, N \geqslant 1 / \delta$,

$$
\varepsilon \geqslant \gamma_{0} \int_{N<|\xi|<2 N} \frac{\left|A_{i}(\xi)\right|^{2}}{\left(1+|\xi|^{2}\right)^{i-1}} d \xi \text { при каждом } i, \quad i=2, \ldots, m,
$$

откуда в силу леммы 7 вытекает требуемое утверждение. Лемма доказана. 
ЛЕмма 10. $L_{2}$-ограниченное решение $u(x, y)$ уравнения (1) обладает свойством

$$
\sup _{0<y<1}\left\|P_{N} u(\cdot, y)\right\|_{L_{2}\left(\mathbb{R}_{n}\right)} \rightarrow 0, \quad N \rightarrow \infty
$$

тогда и только тогда, когда этим свойством обладает кажсдая из функиий $u_{i}(x, y), i=1, \ldots, m$, в равенстве $(35)$ :

$$
\sup _{0<y<1}\left\|P_{N} u_{i}(\cdot, y)\right\|_{L_{2}\left(\mathbb{R}_{n}\right)} \rightarrow 0, \quad N \rightarrow \infty, \quad i=1, \ldots, m .
$$

В силу первого утверждения предыдущей леммы функция $u_{1}(x, y) L_{2}$-ограничена, следовательно, согласно лемме 5 при $N \rightarrow \infty$

$$
\sup _{0<y<1}\left\|P_{N} u_{1}(\cdot, y)\right\|_{L_{2}\left(\mathbb{R}_{n}\right)} \rightarrow 0 .
$$

По лемме 4 аналогичным свойством обладает и функция $U(x, y)$. Поэтому из условия доказываемой леммы вытекает, что

$$
\sup _{0<y<1} \| P_{N}\left(u_{2}(\cdot, y)+\cdots+u_{m}(\cdot, y) \|_{L_{2}\left(\mathbb{R}_{n}\right)} \rightarrow 0, \quad N \rightarrow \infty\right.
$$

т.е. по любому $\varepsilon>0$ найдется такое $N_{0}>R_{2}$, что

$$
\int_{N<|\xi|<2 N}\left|\sum_{i=2}^{m} A_{i}(\xi) w_{i}(\xi, y)\right|^{2} d \xi \leqslant \varepsilon \quad \text { для всех } y \in(0,1),
$$

если только $N \geqslant N_{0}$. Но это неравенство совпадает с неравенством $(57)$, в котором $M^{\prime}=\varepsilon$. Поэтому в силу (61) для любого $i, i=2, \ldots, m$,

$$
\int_{N<|\xi|<2 N} \frac{\left|A_{i}(\xi)\right|^{2}}{\left(1+|\xi|^{2}\right)^{i-1}} \leqslant \frac{\varepsilon}{\gamma_{0}}
$$

для всех достаточно больших $N$. Тогда в силу леммы 7 функции $u_{i}(x, y), i=$ $2, \ldots, m$, имеют нулевой $L_{2}$-предел при $y \rightarrow 0$. После этого доказываемое утверждение вытекает из леммы 8. Лемма доказана.

ЛЕмма 11. $L_{2}$-ограниченное решение $и(x, y)$ уравнения (1) обладает свойством

$$
\sup _{0<y<1}\left\|T_{h} u(\cdot, y)\right\|_{W_{2}^{-1}\left(\mathbb{R}_{n}\right)} \rightarrow 0, \quad|h| \rightarrow 0,
$$

тогда и только тогда, когда этим свойством обладает каждая функиия $u_{i}(x, y), i=1, \ldots, m$, из равенства $(35)$ :

$$
\sup _{0<y<1}\left\|T_{h} u_{i}(\cdot, y)\right\|_{W_{2}^{-1}\left(\mathbb{R}_{n}\right)} \rightarrow 0, \quad|h| \rightarrow 0, \quad i=1, \ldots, m .
$$

Проведя те же рассуждения, что и при доказательстве предыдущей леммы, получим, что функция $u_{1}(x, y)$ обладает свойством $(63)$ и тем самым

$$
\sup _{0<y<1}\left\|T_{h} \sum_{i=2}^{m} u_{i}(\cdot, y)\right\|_{W_{2}^{-1}\left(\mathbb{R}_{n}\right)} \rightarrow 0, \quad|h| \rightarrow 0
$$


т.е. по любому $\varepsilon>0$ найдется такое $\delta=\delta(\varepsilon)>0$, что неравенство

$$
\int_{|\xi|>R_{2}}\left|\sum_{i=2}^{m} A_{i}(\xi) w_{i}(\xi, y)\right|^{2} \frac{\sin ^{4} \frac{(\xi, h)}{2}}{|h|^{2}\left(1+|\xi|^{2}\right)} d \xi \leqslant \varepsilon, \quad y \in(0,1)
$$

имеет место для всех $h,|h| \leqslant \delta$. Это неравенство совпадает с неравенством (56), в котором $M^{\prime}=\varepsilon$, а вместо функций $A_{i}(\xi)$ стоят функции

$$
A_{i}(\xi) \frac{\sin ^{2} \frac{(\xi, h)}{2}}{|h|\left(1+|\xi|^{2}\right)^{1 / 2}}, \quad i=2, \ldots, m
$$

Поэтому в силу рассуждений, которые привели от (56) к (61), сушествует такое $N_{0}$, что имеют место неравенства

$$
\int_{N<|\xi|<2 N} \frac{\left|A_{i}(\xi)\right|^{2} \sin ^{4} \frac{(\xi, h)}{2}}{|h|^{2}\left(1+|\xi|^{2}\right)^{i}} d \xi \leqslant \frac{\varepsilon}{\gamma_{0}}, \quad i=2, \ldots, m
$$

для всех $h,|h| \leqslant \delta$, и всех $N \geqslant N_{0}$.

Далее поступаем, как при доказательстве леммы 8. Проинтегрируем последнее неравенство по единичной сфере $\left\{|h|^{0}=1\right\}$, где $h^{0}=h /|h|$ при $|h| \neq 0$ :

$$
\omega_{n}^{\prime} \int_{N<|\xi|<2 N} \frac{\left|A_{i}(\xi)\right|^{2}}{|h|^{2}\left(1+|\xi|^{2}\right)^{i}} \int_{0}^{1}\left(1-u^{2}\right)^{\frac{n-3}{2}} \sin ^{4}\left(\frac{|\xi||h| u}{2}\right) d u d \xi \leqslant \frac{\varepsilon \omega_{n}}{\gamma_{0}}
$$

$i=2, \ldots, m$, при этом считаем, что $n>1$; случай $n=1$ проше, он так же, как и при доказательстве леммы 8 , исследуется отдельно. Полагая в последнем неравенстве $|h|=1 / N$ (считая при этом, конечно, что $\left.N_{0} \geqslant 1 / \delta\right)$, получим неравенство

$$
\begin{aligned}
\frac{\varepsilon \omega_{n}}{\gamma_{0} \omega_{n}^{\prime}} & \geqslant \int_{N<|\xi|<2 N} \frac{|A(\xi)|^{2} N^{2}}{\left(1+|\xi|^{2}\right)^{i}} \int_{\pi / 6}^{\pi / 3}\left(1-u^{2}\right)^{\frac{n-3}{2}} \sin ^{4}\left(\frac{|\xi| u}{2 N}\right) d u d \xi \\
& \geqslant \frac{27 \sin ^{2} \frac{\pi}{12}}{32 \pi^{2}} \int_{\pi / 6}^{\pi / 3} u^{2}\left(1-u^{2}\right)^{\frac{n-3}{2}} d u \int_{N<|\xi|<2 N} \frac{\left|A_{i}(\xi)\right|^{2}}{\left(1+|\xi|^{2}\right)^{i-1}} d \xi
\end{aligned}
$$

$i=2, \ldots, m$, из которого вытекает, что

$$
\int_{N<|\xi|<2 N} \frac{\left|A_{i}(\xi)\right|^{2}}{\left(1+|\xi|^{2}\right)^{i-1}} d \xi \rightarrow 0 \quad \text { при } N \rightarrow \infty, \quad i=2, \ldots, m
$$

и, следовательно, в силу лемм 7 и 8 имеет место доказываемое утверждение. Лемма доказана.

Докажем, наконец, последнюю лемму, которая позволит из теоремы 2 получить теорему $2^{\prime}$. 
Лемма 12. Для всякой функиии и $(x, y)$ из $C\left((0,1] ; L_{2}\left(\mathbb{R}_{n}\right)\right)$ условие

$$
\sup _{0<y<1}\left\|T_{h} u(\cdot, y)\right\|_{W_{2}^{-1}\left(\mathbb{R}_{n}\right)} \rightarrow 0 \quad n p u \quad h \rightarrow 0 \quad\left(h \in \mathbb{R}_{n}\right)
$$

әквивалентно условиям

$$
\sup _{0<y<1}\left\|T_{h_{k} i_{k}} u(\cdot, y)\right\|_{W_{2}^{-1}\left(\mathbb{R}_{n}\right)} \rightarrow 0 \quad \text { npu } \quad h_{k} \rightarrow 0 \quad\left(h_{k} \in \mathbb{R}_{1}\right), \quad k=1, \ldots, n
$$

$\left(\right.$ напомним, что $\left.i_{1}=(1, \ldots, 0), \ldots, i_{n}=(0, \ldots, 0,1), h=h_{1} i_{1}+\cdots+h_{n} i_{n}\right)$.

В силу формулы (9) имеем равенство

$$
\left\|T_{h} u(\cdot, y)\right\|_{W_{2}^{-1}\left(\mathbb{R}_{n}\right)}^{2}=4 \int_{\mathbb{R}_{n}} \frac{|\widetilde{u}(\xi, y)|^{2}}{\left(1+|\xi|^{2}\right)|h|^{2}} \sin ^{4} \frac{(\xi, h)}{2} d \xi
$$

Так как

$$
\begin{aligned}
\sin \frac{(\xi, h)}{2}= & \sin \left(\frac{\xi_{1} h_{1}}{2}+\cdots+\frac{\xi_{n} h_{n}}{2}\right)=\sin \frac{\xi_{1} h_{1}}{2} \cos \left(\frac{\xi_{2} h_{2}}{2}+\cdots+\frac{\xi_{n} h_{n}}{2}\right) \\
& +\cos \frac{\xi_{1} h_{1}}{2} \sin \frac{\xi_{2} h_{2}}{2} \cdot \cos \left(\frac{\xi_{3} h_{3}}{2}+\cdots+\frac{\xi_{n} h_{n}}{2}\right) \\
& +\cdots+\cos \frac{\xi_{1} h_{1}}{2} \cdots \cos \frac{\xi_{n-1} h_{n-1}}{2} \sin \frac{\xi_{n} h_{n}}{2},
\end{aligned}
$$

TO

$$
\left|\sin \frac{(\xi, h)}{2}\right| \leqslant\left|\sin \frac{\xi_{1} h_{1}}{2}\right|+\left|\sin \frac{\xi_{2} h_{2}}{2}\right|+\cdots+\left|\sin \frac{\xi_{n} h_{n}}{2}\right| .
$$

Согласно неравенству Гёльдера

$$
\sin ^{4} \frac{(\xi, h)}{2} \leqslant n^{3}\left(\sin ^{4} \frac{\xi_{1} h_{1}}{2}+\cdots+\sin ^{4} \frac{\xi_{n} h_{n}}{2}\right)
$$

поэтому

$$
\begin{aligned}
\left\|T_{h} u(\cdot, y)\right\|_{W_{2}^{-1}\left(\mathbb{R}_{n}\right)}^{2} & \leqslant 4 n^{3} \sum_{k=1}^{n} \int_{\mathbb{R}_{n}} \frac{|\widetilde{u}(\xi, y)|^{2}}{\left(1+|\xi|^{2}\right)|h|^{2}} \sin ^{4} \frac{\xi_{k} h_{k}}{2} d \xi \\
& \leqslant 4 n^{3} \sum_{k=1}^{n} \int_{\mathbb{R}_{n}} \frac{|\widetilde{u}(\xi, y)|^{2}}{1+|\xi|^{2}} \frac{\sin ^{4} \frac{\xi_{k} h_{k}}{2}}{h_{k}^{2}} d \xi \\
& \leqslant 4 n^{3} \sum_{k=1}^{n} \sup _{0<y<1}\left\|T_{h_{k} i_{k}} u(\cdot, y)\right\|_{W_{2}^{-1}\left(\mathbb{R}_{n}\right)}^{2}
\end{aligned}
$$

Из этого неравенства вытекает, что из (11') следует (11). Обратное очевидно. Лемма доказана. 
ДокаЗАТЕЛЬСтво теорем 1 и 2. Докажем сначала утверждения о необходимости. Если функция $u(x, y)$ имеет $L_{2}$-предел при $y \rightarrow 0$, т.е. если $u(x, y) \in C\left([0,1] ; L_{2}\left(\mathbb{R}_{n}\right)\right)$, то она, конечно, $L_{2}$-ограничена, т.е. вьполняется условие (2), и в силу леммы 4 она удовлетворяет условиям (10) и (11).

Докажем теперь утверждения о достаточности. Из условия ограниченности (2) в силу леммы 9 имеем $L_{2}$-ограниченность каждой функции $u_{i}(x, y), i=1, \ldots, m$, в равенстве $(35)$, а тогда в силу леммы 5 функция $u_{1}(x, y)$ имеет $L_{2}$-предел при $y \rightarrow 0$. Из условий $(10)$ и (11) согласно леммам 10 и 11 получаем, что условиям (10) и (11) удовлетворяет каждое из оставшихся слагаемых $u_{i}(x, y), i=2, \ldots, m$, в формуле (35). Но тогда согласно лемме 8 каждая функция $u_{i}(x, y), i=2, \ldots, m$, имеет (нулевой) $L_{2}$-предел при $y \rightarrow 0$. Таким образом, функция $u(x, y)$ имеет $L_{2}$-предел при $y \rightarrow 0$. Теоремы доказаны.

Доказательство теоремы $2^{\prime}$ получается немедленно из теоремы 2 , если принять во внимание лемму 12.

\section{Список литературы}

1. Михайлов В. П. О существовании предельных значений решений полигармонического уравнения на границе области // Матем. сб. 1996. Т. 187. № 11. С. 89-114.

2. Векуа И. Н. Новые методы решения эллиптических уравнений. М.-Л.: Гостехиздат, 1948.

3. Ройтберг Я. А. О предельных значениях по поверхностям, параллельньм границе, обобщенных решений эллиптических уравнений // Докл. АН СССР. 1978. Т. 238. №6. C. $1303-1306$.

4. Михайлов В.П. Об одном достаточном условии существования предельных значений полигармонических функций на границе области // Труды 2-го международного семинара "Дифференциальные уравнения и их приложения". Самара: Изд-во СГУ, 1998. C. $115-121$.

Математический институт

им. В.А. Стеклова РАН

Поступила в редакцию

17.03 .1999 